\title{
The origin of the anomalous intensity ratio between very bright UV Fell lines and their satellites in gaseous condensations close to the star $\eta$ Carinae
}

\author{
V. Klimov ${ }^{1}$, S. Johansson ${ }^{2}$, and V. S. Letokhov ${ }^{2,3}$ \\ 1 P.N. Lebedev Physical Institute, Russian Academy of Sciences, 53 Leninsky Prospect, Moscow 117294, Russia \\ 2 Atomic Astrophysics, Lund Observatory, Lund University, PO Box 43, Lund 221 00, Sweden \\ 3 Institute of Spectroscopy, Russian Academy of Sciences, Troitsk 142190, Moscow region, Russia \\ e-mail: vladilen.letokhov@astro.lu.se
}

Received 12 July 2001 / Accepted 14 January 2002

\begin{abstract}
We present a model that explains the anomalous intensity ratios between each of two very bright UV lines of Fe II at $2507 \AA$ and $2509 \AA$ and its corresponding satellite line, observed in HST spectra of a compact gaseous condensation (blob B) in the vicinity of the star $\eta$ Car. The model is based on the assumption that the FeII transitions have a substantial optical thickness, which varies as a result of the photodepletion of the long-lived lower states, $c^{4} \mathrm{~F}_{7 / 2,9 / 2}$, by the intense Ly $\alpha$ radiation. In conditions where the photodepletion rates of these two states differ, the resonance scattering lengths of the spectral lines become different as well. In the presence of a weak, nonresonant (continuous) absorption, this gives rise to a difference in attenuation between the spectral lines (the Hummer effect (Hummer 1968)). It is the combination of these two effects that explains the anomalous (as compared with laboratory data) intensity ratios observed in the two pairs of UV FeII lines. Ionization channels of stimulated photodepletion by the Ly $\alpha$ radiation, including the possible role of autoionization levels, are considered.
\end{abstract}

Key words. atomic processes - line: formation - radiation mechanisms: non-thermal - stars: individual: $\eta$ Carinae

\section{Introduction. Problem}

One of the puzzles discovered in the course of highspectral-and-spatial-resolution observations of the star $\eta$ Carinae with the Hubble Space Telescope was anomalous (other than predicted by laboratory data) intensity ratios between two very bright narrow FeII lines at $2507 \AA$ and $2509 \AA$ and their satellites. This observation was so extraordinary that it even gave rise to the assumption of stimulated amplification of these lines (Johansson et al. 1996). In a recent report (Johansson \& Letokhov 2001b), we have analyzed a number of photoprocesses induced by the intense Ly $\alpha$ radiation in a gaseous blob (blob B, see below) in the immediate vicinity of the star $\eta$ Car. These processes can, in principle, help to qualitatively describe all these anomalous effects within the framework of the physical parameters estimated for $\eta$ Car and its blobs (Davidson \& Humphreys 1971; Davidson 1999; Hamann et al. 1999). Using all these facts as the base, we have considered (Johansson \& Letokhov 2001b) a three-zone blob model including an active HII region (which generates Ly $\alpha$ photons), an intermediate passive HI region

Send offprint requests to: S. Johansson, e-mail: sveneric.johansson@astro.lu.se (which broadens the Ly $\alpha$ spectral line because of Doppler diffusion), and a passive HI + FeII region, in which the broadened Ly $\alpha$ radiation can excite FeII ions and thus cause the abnormal UV fluorescence observed. Moreover, this model predicts a significant optical thickness for the FeII transitions at $\lambda \lambda 2507,2509$, which can explain the anomalous intensity ratio between these bright UV lines and their satellites. In the present paper, we develop this model further, so that this anomaly can be explained without the need to exploit the idea of stimulated amplification of the corresponding transitions.

Before going on to the main subject of this paper, let us formulate the main features of the model presented earlier (Johansson \& Letokhov 2001a,b) of the origin of the extremely bright UV lines of FeII in the gaseous condensations near the star $\eta$ Carinae.

\section{A model of the origin of the extremely bright UV Fell lines}

According to observational data (Davidson \& Humphreys 1997), the Weigelt blobs (Weigelt \& Ebersberger 1986) close to $\eta$ Car (for example, blob B) are compact gas condensations (low-intensity compact ejecta with a high 
hydrogen concentration $N_{\mathrm{H}} \simeq 10^{6} \mathrm{~cm}^{-3}$ and a Fe concentration of $N_{\mathrm{Fe}} \simeq 10^{2} \mathrm{~cm}^{-3}$ ).

The blob diameter is $D \leq 10^{15} \mathrm{~cm}$, and the distance from the central star is $R_{\mathrm{b}} \simeq 3 \times 10^{16} \mathrm{~cm}$. The diameter of the photosphere of the central star is $2 r_{\mathrm{s}}=$ $d_{\mathrm{s}} \simeq 3 \times 10^{13} \mathrm{~cm}$, and its effective temperature is $T_{\mathrm{s}}=$ $(20-30) \times 10^{3} \mathrm{~K}$. These parameter values are preliminary. For example, according to recent HST/STIS observations (T. Gull, private communication, April 2001), the distance $R_{\mathrm{b}}$ between the blob $\mathrm{B}$ and the central star is around $3 \times 10^{15} \mathrm{~cm}$, i.e. an order of magnitude smaller than derived from previous data. The fact that the blob is located close enough to the central star is of importance for our model. The dilution factor of the radiation from $\eta$ Car, $\Omega \cong \frac{1}{4}\left(r_{\mathrm{s}} / R_{\mathrm{b}}\right)^{2}$, is around $10^{-6}-10^{-5}$, which is many orders of magnitude greater than that for a typical planetary nebula. For illustration, we present in Fig. 1 a schematic picture of a gas condensation having a spherical shape with an average diameter $D$ (as judged from density) and an average volume concentration $N_{\mathrm{H}}$ of hydrogen atoms and ions and being located at the distance $R_{\mathrm{b}} \gg D$ from the star. The most intense part of the stellar radiation with $\mathrm{h} \nu>\mathrm{h} \nu_{\mathrm{c}}=13.6 \mathrm{eV}$ is absorbed almost completely by neutral hydrogen having a concentration of $N_{\mathrm{HI}}\left(N_{\mathrm{HI}} \ll N_{\mathrm{HII}} \approx N_{\mathrm{H}}\right)$, provided that the optical density $\tau\left(\nu_{\mathrm{c}}\right)$ of the Lyman-continuum is high enough, i.e.,

$\sigma_{\mathrm{ph}}\left(\nu_{\mathrm{c}}\right) N_{\mathrm{HI}} D \gg 1$

Condition (1) is satisfied if the hydrogen density $N_{\mathrm{H}}$ exceeds some critical value $N_{\mathrm{H}}^{\mathrm{cr}}$ :

$N_{\mathrm{H}}^{\mathrm{cr}}=\left(\frac{I_{\mathrm{ph}}}{\alpha D}\right)^{1 / 2}=\frac{r_{\mathrm{s}}}{R_{\mathrm{b}}}\left(\frac{P\left(\nu_{\mathrm{c}}, T_{\mathrm{s}}\right) \Delta \nu_{\mathrm{ph}}}{\alpha D}\right)^{1 / 2}$,

where $\alpha$ is the rate coefficient of the $\mathrm{HII} \rightarrow \mathrm{HI}$ recombination process, and $I_{\mathrm{ph}}$ is the effective intensity of the radiation incident on the blob surface and ionizing hydrogen. As an estimate we use, as in our previous paper (Johansson \& Letokhov 2001b), average values of the blackbody radiation intensity $P\left(\nu_{\mathrm{c}}\right)$ and photoionization cross section $\sigma_{\mathrm{ph}}(\nu)$ in the effective frequency interval $\Delta \nu_{\mathrm{ph}} \simeq 3 \mathrm{eV}$ above $\nu_{\mathrm{c}}$, where $P(\nu)$ and $\sigma_{\mathrm{ph}}(\nu)$ are sufficiently large. Condition (1) means that the local Strömgren boundary separating the HII and HI regions is inside the gas condensation being considered, as shown in Fig. 1. For blob B near $\eta$ Carinae at $D \leq 10^{15} \mathrm{~cm}$ and $T_{\mathrm{s}}=30000 \mathrm{~K}$, the critical density $N_{\mathrm{H}}^{\mathrm{cr}} \geq 10^{8} \mathrm{~cm}^{-3}$.

The physical depth $l_{\mathrm{ph}}$ at which the photoionization state of hydrogen is sustained at $N_{\mathrm{H}}>N_{\mathrm{H}}^{\mathrm{cr}}$ is defined by the expression

$\frac{l_{\mathrm{ph}}}{D} \simeq\left(\frac{N_{H}^{\mathrm{cr}}}{N_{\mathrm{H}}}\right)^{2}=\frac{1}{N_{\mathrm{H}}^{2}} \frac{I_{\mathrm{ph}}}{\alpha D}$

At $N_{\mathrm{H}} \gg N_{\mathrm{H}}^{\mathrm{cr}}$, the depth $l_{\mathrm{ph}} \ll D$, i.e. there is a thin front layer of the blob facing the central star (Fig. 1).
At $N_{\mathrm{H}}>N_{\mathrm{H}}^{\mathrm{cr}}$ the neutral component HI absorbs almost all of the power $\mathcal{P}$ (in photons/s) of the Lymancontinuum radiation coming from the star:

$$
\begin{aligned}
\mathcal{P}_{\mathrm{abs}}\left(\nu>\nu_{c}\right) & \simeq \Omega_{0} S_{\mathrm{abs}} \int_{\nu_{\mathrm{c}}}^{\infty} P\left(\nu, T_{\mathrm{s}}\right) \mathrm{d} \nu \\
& \simeq \Omega \pi^{2} D^{2} \frac{P\left(\nu_{\mathrm{c}}, T_{\mathrm{s}}\right)}{P} \Delta \nu_{\mathrm{ph}},
\end{aligned}
$$

where $\Omega_{0}=4 \pi \Omega$ is the solid angle and $S_{\text {abs }}=\frac{\pi}{4} D^{2}$ is the disc area of the blob absorbing the incident radiation from the central star. Most of the absorbed energy is reemitted due to radiative recombination of the hydrogen ions formed. The major proportion $(\eta \approx 0.7)$ of the absorbed energy is emitted in the resonance line HI Ly $\alpha$. The maximum value of the optical density $\tau_{0}(\mathrm{Ly} \alpha)$ with reference to the center of $\operatorname{Ly} \alpha$ in the HII region is

$\tau_{0}^{\mathrm{m}} \simeq \frac{\sigma_{0}}{\sigma_{\mathrm{ph}}\left(\nu_{\mathrm{c}}\right)} \simeq 5 \times 10^{3}$,

where we have set $\sigma_{0} \simeq 1.4 \times 10^{-14} \mathrm{~cm}^{2}$ and $\sigma_{\mathrm{ph}}\left(\nu_{\mathrm{c}}\right) \simeq$ $3 \times 10^{-18} \mathrm{~cm}^{2}$ (average value) in the frequency interval $\left(\nu_{\mathrm{c}}, \nu_{\mathrm{c}}+\Delta \nu_{\mathrm{ph}}\right)$. For illustration, we show in Fig. 2 the qualitative distribution of the average concentrations of HII and HI inside the blob, as well as the evolution of the Ly $\alpha$ radiation spectrum in various zones of the blob.

The Ly $\alpha$ radiation proves to be trapped in the HII region, but the diffusion confinement time is limited by the fact that the number of scattering events is limited because of the Doppler frequency redistribution of the scattered Ly $\alpha$ photons. This redistribution makes them leave the trapping region relatively fast via the wings of the Doppler profile (Osterbrock 1967). Since the optical density $\tau_{0}$ is limited to $\tau_{0}^{\mathrm{m}}=\left(\sigma_{0} / \sigma_{\mathrm{ph}}\right)$, and the damping factor for $\operatorname{Ly} \alpha$ is $a=\delta \nu_{\mathrm{rad}} / \delta \nu_{\mathrm{D}} \simeq 4 \times 10^{-4}$, the optical density in the Lorentz wings is $a \tau_{0}^{\mathrm{m}} \simeq 1$, where we assume the Doppler spectral width to be $\delta \nu_{\mathrm{D}} \simeq 6 \mathrm{~cm}^{-1}$ and the natural spectral width, $\delta \nu_{\mathrm{rad}} \simeq 2.5 \times 10^{-3} \mathrm{~cm}^{-1}$. Therefore, the Ly $\alpha$ photons leave the HII region by diffusion while suffering an increase in the Doppler width $\Delta \nu_{\mathrm{D}}$ by a factor of $\left(\ln \tau_{0}^{\mathrm{m}}\right)^{1 / 2} \approx 3$, so that the total power emitted by the surface of the blob into Ly $\alpha$ is

$\mathcal{P}_{\mathrm{em}}(\mathrm{Ly} \alpha) \simeq 4 \pi S_{\mathrm{em}} P\left(\nu_{\mathrm{Ly} \alpha}, T_{\mathrm{br}}\right)\left(\Delta \nu_{\mathrm{D}} \sqrt{\ln \tau_{0}^{\mathrm{m}}}\right)$,

where $S_{\mathrm{em}}=\pi D^{2}$ is the area of the emitting surface of the spherical blob and $P\left(\nu_{\mathrm{Ly} \alpha}, T_{\mathrm{br}}\right)$ is the average spectral intensity of $\operatorname{Ly} \alpha$ on the blob surface, given by the Planck distribution at a frequency of $\nu_{\mathrm{Ly} \alpha}$ and a spectral brightness temperature of $T_{\mathrm{br}}$, account being taken of the spectral line broadening due to trapping. In the steadystate case where the absorption of the trapped Ly $\alpha$ in the HII region of the blob is negligible, we have

$\eta \mathcal{P}_{\text {abs }}\left(\nu>\nu_{\mathrm{c}}\right)=\mathcal{P}_{\text {em }}(\operatorname{Ly} \alpha)$

It follows from (7) that the brightness temperature $T_{\mathrm{br}}$ of the Ly $\alpha$ radiation on the open surface of the blob can be estimated by the expression

$\left.P\left(\nu_{\mathrm{Ly} \alpha}, T_{\mathrm{br}}\right) \simeq \frac{\eta}{4} \Omega \frac{\Delta \nu_{\mathrm{ph}}}{\Delta \nu_{\mathrm{D}} \sqrt{\ln \tau_{0}^{\mathrm{m}}}} \overline{P\left(\nu_{\mathrm{c}}, T_{\mathrm{s}}\right.}\right)$. 


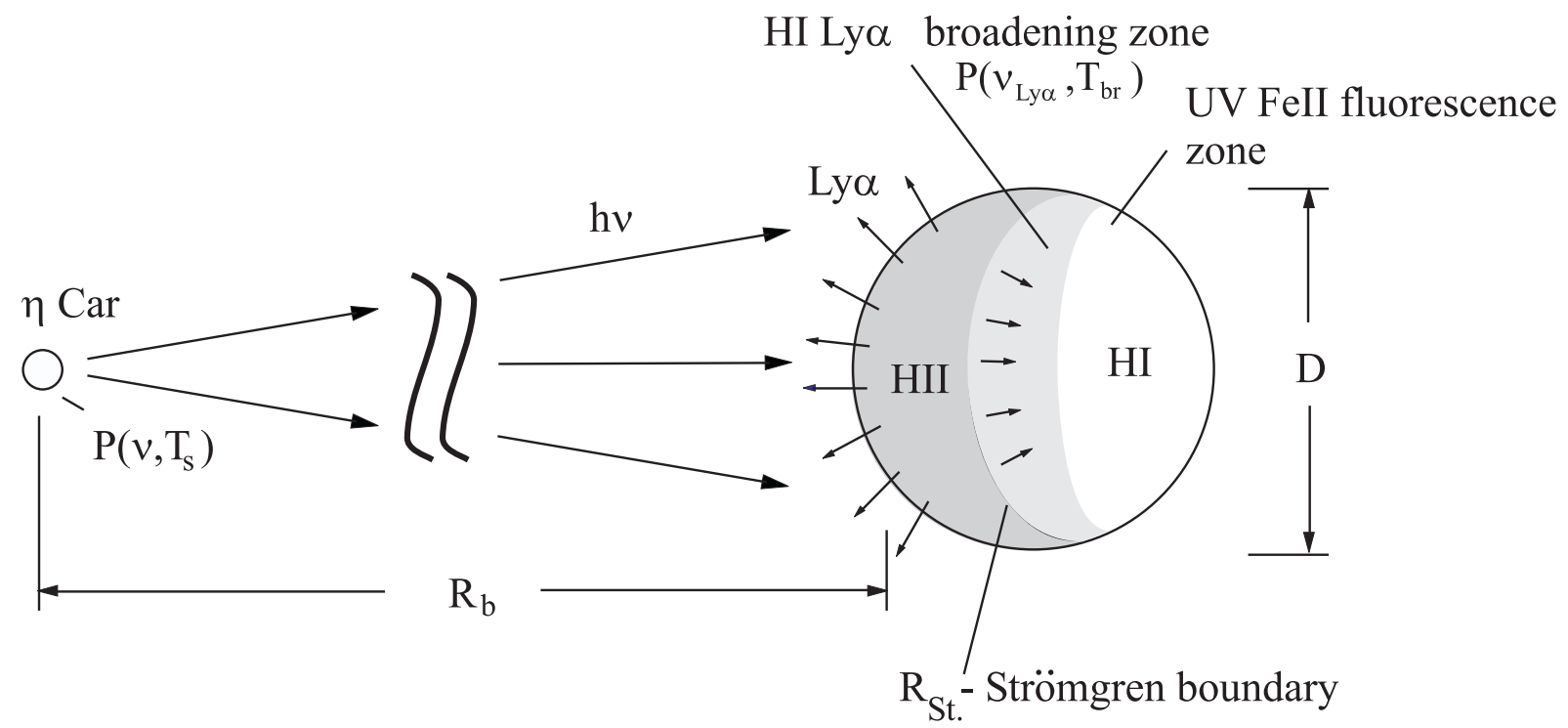

Fig. 1. Model geometry of the compact gas condensation (blob) in the vicinity of the central star of $\eta$ Carinae.

It can be seen that the dilution factor $\Omega$ can largely be compensated for by the effect of the spectral compression of the absorbed energy into the relatively narrow Ly $\alpha$ radiation. Note that the intensity of the Ly $\alpha$ radiation inside the HII region of the blob is higher than that on the blob surface, $P\left(\nu_{\mathrm{Ly} \alpha}, T_{\mathrm{br}}\right)$, by a factor of $\ln \tau_{0}^{\mathrm{m}}$ due to diffusion confinement. Therefore, to determine the brightness temperature $\tilde{T}_{\mathrm{br}}$ of the radiation incident on the boundary between the HII and HI regions inside the blob (the Strömgren boundary in Fig. 1), the right-hand side of expression (7) should be increased by $\ln \tau_{0}^{\mathrm{m}} \simeq 7$ times, which is equivalent to the same increase in $\Omega$ :

$\left.P_{\text {St.b }}\left(\nu_{\mathrm{Ly} \alpha}, \tilde{T}_{\mathrm{br}}\right) \simeq \frac{\eta}{4} \Omega \frac{\Delta \nu_{\mathrm{ph}} \sqrt{\ln \tau_{0}^{\mathrm{m}}}}{\Delta \nu_{\mathrm{D}}} \overline{P\left(\nu_{\mathrm{c}}, T_{\mathrm{s}}\right.}\right)$.

The temperature $\tilde{T}_{\mathrm{br}}$ is fairly close to the temperature of the blackbody radiation of the central star's photosphere (for $\Omega \simeq 10^{-6}-10^{-5}$, the temperature $\tilde{T}_{\mathrm{br}}$ is $\left.(10-20) \times 10^{3} \mathrm{~K}\right)$, but the spectral width of the line radiation leaving the active HII region is still insufficient to compensate for the difference in wavelength between the Ly $\alpha$ emission and the FeII absorption lines (see the top part of Fig. 2). This radiation suffers further spectral diffusion both in the HII/HI transition region (the Strömgren boundary), which is optically denser for $\operatorname{Ly} \alpha$, and mainly in the passive, weakly ionized HI region.

The physical depth $\delta l_{\mathrm{ph}}$ of the HII/HI transition region is shallow:

$\delta l_{\mathrm{ph}} \simeq \frac{1}{\sigma_{\mathrm{ph}} N_{\mathrm{H}}} \ll l_{\mathrm{ph}}$,

in comparison with the physical length $l_{\mathrm{ph}}$ of the photoionization depth (the physical depth of the active HII part of blob B):

$l_{\mathrm{ph}} \simeq \delta l_{\mathrm{ph}} \frac{N_{\mathrm{HII}}}{N_{\mathrm{HI}}} \leq D$, defined by expression (3). Note that, for the reader's convenience, we present here these well-known expressions (Mihalas 1978) in order to give explanations to the designations used. The optical thickness of the HII/HI transition layer with physical width $\delta l_{\mathrm{ph}}$ can be estimated as:

$\tau_{0}^{\mathrm{tr}} \simeq N_{\mathrm{HI}}^{\mathrm{tr}} \delta l_{\mathrm{ph}} \sigma_{0} \simeq \frac{1}{2} \frac{\sigma_{0}}{\sigma_{\mathrm{ph}}} \simeq 2 \times 10^{3}$

where $N_{\mathrm{HI}}^{\mathrm{tr}} \simeq \frac{1}{2} N_{\mathrm{H}}$ is the average concentration of $\mathrm{HI}$ in the transition layer, and $\delta l_{\mathrm{ph}}$ is determined by Eq. (10). The high density of HI compensates the small width of this layer and as a result the optical thickness of the transient layer $\tau_{0}^{\mathrm{tr}}$ is almost equal to the optical thickness $\tau_{0}^{\mathrm{m}}$ of the whole active zone. Nevertheless, these values of $\tau_{0}$ are much smaller than the magnitude required for the $\operatorname{Ly} \alpha$ excitation of FeII:

$\tau_{0}^{\mathrm{exc}}=\sigma_{0} N_{\mathrm{H}} D=\frac{\sigma_{0}}{\sigma(\Delta \nu)}=\frac{(2 \Delta \nu)^{2}}{\delta \nu_{\mathrm{rad}} \delta \nu_{\mathrm{D}}} \simeq 7 \times 10^{6}$.

However, the remaining, dissipating (passive) volume of the blob is also large enough to provide for $\tau_{0}^{\text {exc }} \simeq 10^{7}$ and the appropriate broadening of the Ly $\alpha$ line as a result of the Doppler frequency diffusion of the radiation on the Lorentz wing of the Ly $\alpha$ resonance line.

The physical depth $l_{\mathrm{br}}$ of the passive HI region, at which the diffusion spectrum broadening necessary for the excitation of FeII is reached, is defined by the expression

$l_{\mathrm{br}} \simeq \frac{1}{\Delta \nu N_{\mathrm{H}}} \simeq \frac{\tau_{0}^{\mathrm{exc}}}{\sigma_{0} N_{\mathrm{H}}}$

The depth $l_{\text {br }}$ (see Figs. 1 and 2) can be compared with the depth $l_{\mathrm{ph}}$ of the photoionization region. Their ratio is given by

$\frac{l_{\mathrm{br}}}{l_{\mathrm{ph}}} \simeq \frac{\tau_{0}^{\mathrm{exc}}}{\sigma_{0} N_{\mathrm{H}} D}\left(\frac{N_{\mathrm{H}}}{N_{\mathrm{cr}}}\right)^{2}$. 


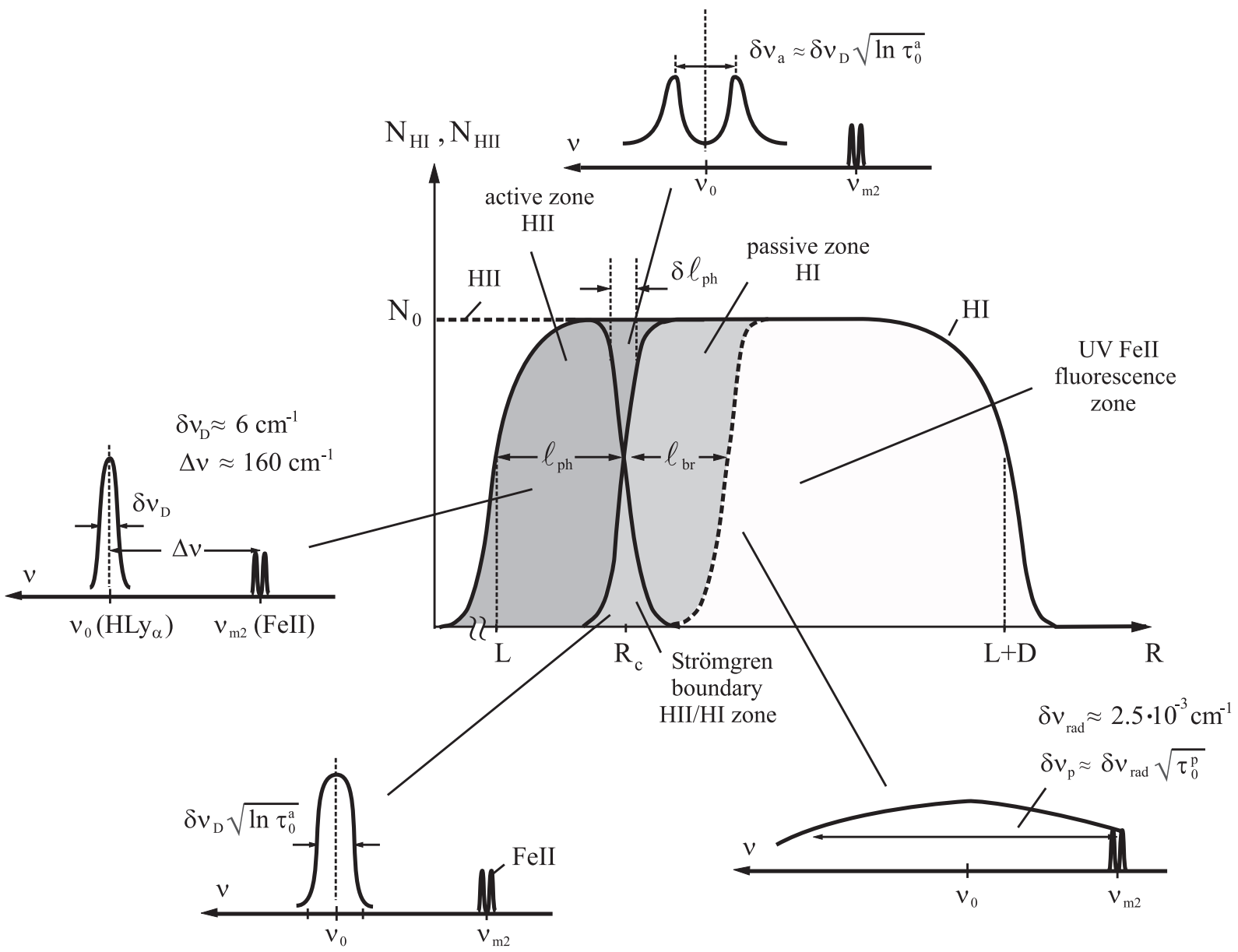

Fig. 2. Radial profiles of the ionized $\left(N_{\mathrm{HII}}\right)$ and neutral hydrogen $\left(N_{\mathrm{HI}}\right)$ concentrations and a qualitative evolution of the spectral shape of the Ly $\alpha$ radiation in the active HII region, the Strömgren boundary region, and the passive fluorescence region relative to the FeII absorption profile.

At $N_{\mathrm{H}}=N_{\mathrm{cr}}$ the depth $l_{\mathrm{br}} \simeq(0.01$ to 0.1$) l_{\mathrm{ph}}$, so that the transition region fully provides for the necessary diffusion broadening of Ly $\alpha$. At $N_{\mathrm{H}}=10 N_{\mathrm{cr}}$, the depth $l_{\mathrm{br}}$ increases to (0.1 to 1$) l_{\mathrm{ph}}$. Finally, $l_{\mathrm{br}} \simeq l_{\mathrm{ph}}$ at a hydrogen concentration in the gas condensation defined as

$N_{\mathrm{H}} \simeq \frac{\sigma_{0} I_{\mathrm{ph}}}{\alpha \tau_{0}^{\mathrm{exc}}}$.

For example, for blob B in the vicinity of $\eta$ Car, this regime is reached at $N_{\mathrm{H}} \simeq 10^{8}-10^{9} \mathrm{~cm}^{-3}$.

The following two well-known processes take place in the passive HI region where the Ly $\alpha$ radiation is transferred in a medium with $\tau_{0}^{\text {exc }} \gg \tau_{0}^{\mathrm{m}}$. At first, a more effective increase of the radiation spectral width (in proportion to $\sqrt{\tau_{0}}$ ) occurs as a result of the Doppler frequency redistribution in the Lorentz wings, which compensates, according to (12), for the frequency difference $\Delta \nu$ between the Ly $\alpha$ and the FeII absorption lines. The diffusion broadening of the Ly $\alpha$ radiation spectrum in the HI region of the blob is limited exactly by the photoselective absorption of this radiation by FeII at $\lambda=1218 \AA$ and intensity transformation in the blue wing of Ly $\alpha$ into an intense UV fluorescence of FeII (see the bottom right- hand part of Fig. 2). Secondly, a more effective diffusive confinement of the Ly $\alpha$ radiation takes place, resulting in a substantial increase, by at least $\left(\Delta \nu / \Delta \nu_{\mathrm{D}}\right) \simeq 50$ times, of its spectrum-integrated intensity. However, to carry out a quantitative analysis of the radiation transfer in the HI-to-HII boundary region, in which the HI and HII concentrations change sharply over a short distance of $l_{\mathrm{tr}} \simeq\left(\sigma_{\mathrm{tr}} N_{\mathrm{H}}\right)^{-1} \simeq 10^{9}-10^{10} \mathrm{~cm} \ll D$ requires a special computer modeling, and this will be the subject of a special publication. So, in the case of blob B near $\eta$ Car, the extreme spectral brightness of the Ly $\alpha$ radiation causes a very strong excitation of FeII ions contained in the blob matter as a result of the Ly $\alpha$ quasi-coincidence $(\Delta \lambda \simeq 3 \AA)$ with the absorption line of FeII in a low-lying metastable state. The subsequent extremely bright fluorescence on UV FeII lines ensures an effective radiative cooling of the blob.

To conclude this brief consideration of the evolution of the spectrum and intensity of the Ly $\alpha$ radiation, let us emphasize that Ly $\alpha$ has a very high optical density, so that one should take into account the diffusive frequency redistribution far in the Lorentz wings. At the same time the transfer of the UV FeII radiation (considered in Sect. 5 


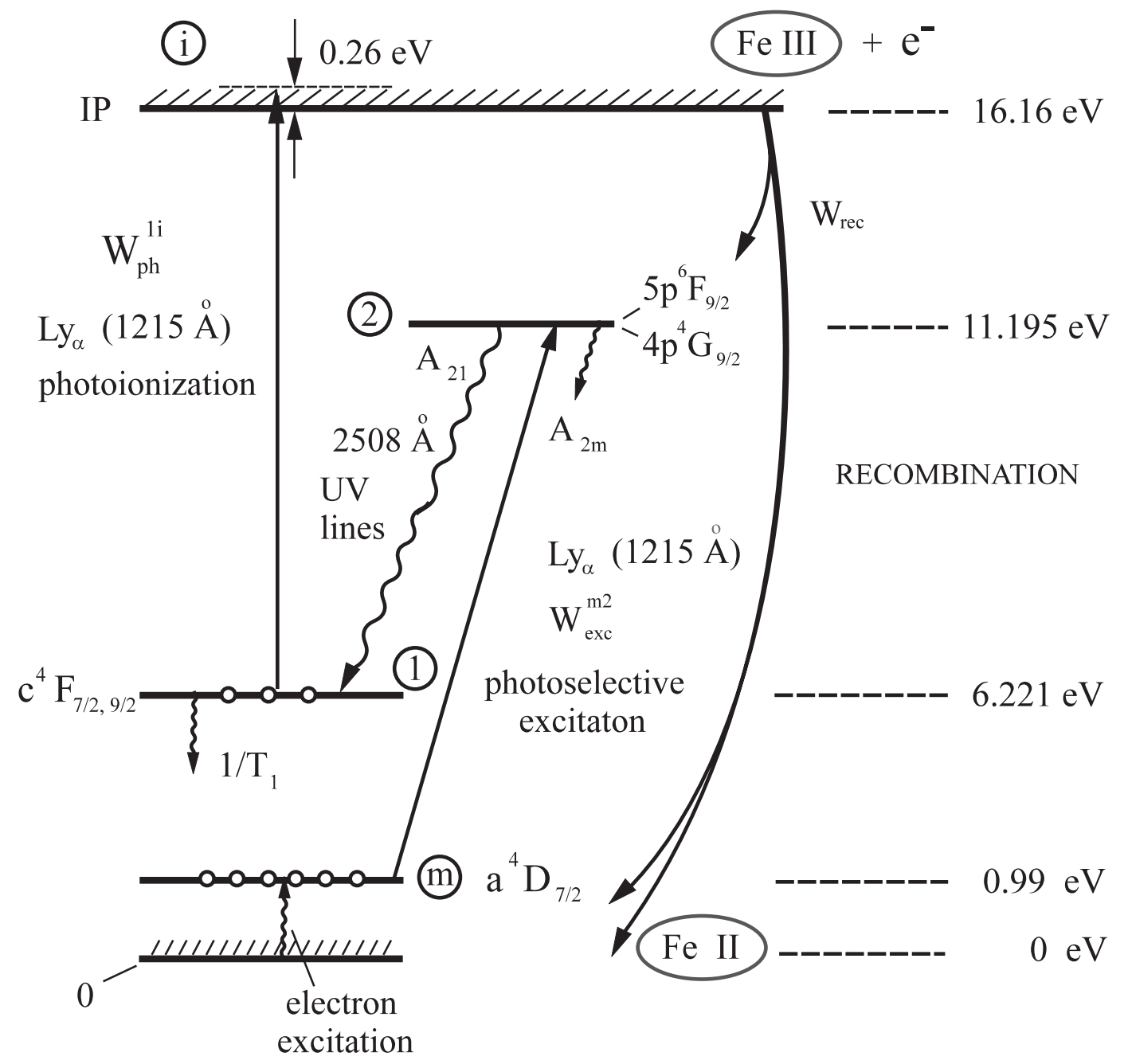

Fig. 3. Energy levels and quantum transitions in FeII relevant to the anomalous UV FeII spectral lines.

below) takes place at a more moderate optical density $\tau(\mathrm{UVF}$ II $) \leq 2 \times 10^{2}$, so that the Lorentz wings take no part in the evolution of the intensity and spectrum of UV FeII. This remark is specially made to avoid confusion between similar designations for the two different spectral lines, because in both cases (for the Ly $\alpha$ and the UV FeII line) the transfer of radiation in an optically dense medium plays an important part.

\section{Anomalous intensity ratio between the UV Fell lines and their satellites}

A schematic diagram of the FeII quantum states, radiative transitions, and notations involved in the present problem is shown in Fig. 3. The low-lying metastable state $a^{4} D_{7 / 2}$ ( $m$-state) with the energy $E_{\mathrm{m}}=0.99 \mathrm{eV}$ is one of the low-lying metastable states in FeII. The wavelength of the broad HLy $\alpha$ line coincides with that of the absorption lines $\mathrm{a}^{4} \mathrm{D}_{7 / 2} \rightarrow 5 \mathrm{p}^{6} \mathrm{~F}_{9 / 2}, 4 \mathrm{p}^{4} \mathrm{G}_{9 / 2}(m \rightarrow 2$ allowed transition). The radiative transitions from levels 2 down to the long-lived states $c^{4} \mathrm{~F}_{9 / 2}, c^{4} \mathrm{~F}_{7 / 2}(2 \rightarrow 1$ transitions $)$ include two strong lines ( $\mathrm{A}$ and $\mathrm{C}$ ), which terminate on the $c^{4} \mathrm{~F}_{7 / 2}$ level, and two weak lines (b and $\mathrm{d}$ ), which terminate on the $c^{4} \mathrm{~F}_{9 / 2}$ level. These spectral lines have anomalous intensities.

For illustration, we present in Fig. 4 spectra from blob B of the UV lines observed with the Hubble Space Telescope (HST) and synthetic spectra based on laboratory intensities (Johansson \& Zethson 1999). As can be seen, the weak satellite lines ( $b$ and $d$ ) practically vanish in the spectra of blob B. The simultaneous spectralselective attenuation of two close spectral lines seems to have an extremely small probability. On the other hand, the anomalous intensity ratio observed between the strong UV spectral lines and their satellites (left-hand part of Fig. 4) can potentially be explained by amplification as a result of stimulated $2 \rightarrow 1$ transitions (Johansson et al. 1996). It is possible if the stimulated emission rate $W_{21}^{\text {st.em. }}$ is higher than the stimulated absorption rate $W_{12}^{\text {st.abs }}$ :

$W_{21}^{\text {st.em. }}>W_{12}^{\text {st.abs. }}$

i.e., in the case of population inversion:

$\frac{N_{2}}{g_{2}}>\frac{N_{1}}{g_{1}}$. 


\section{Fe II $2507 \& 2509 \AA$ in the Weigelt blobs}

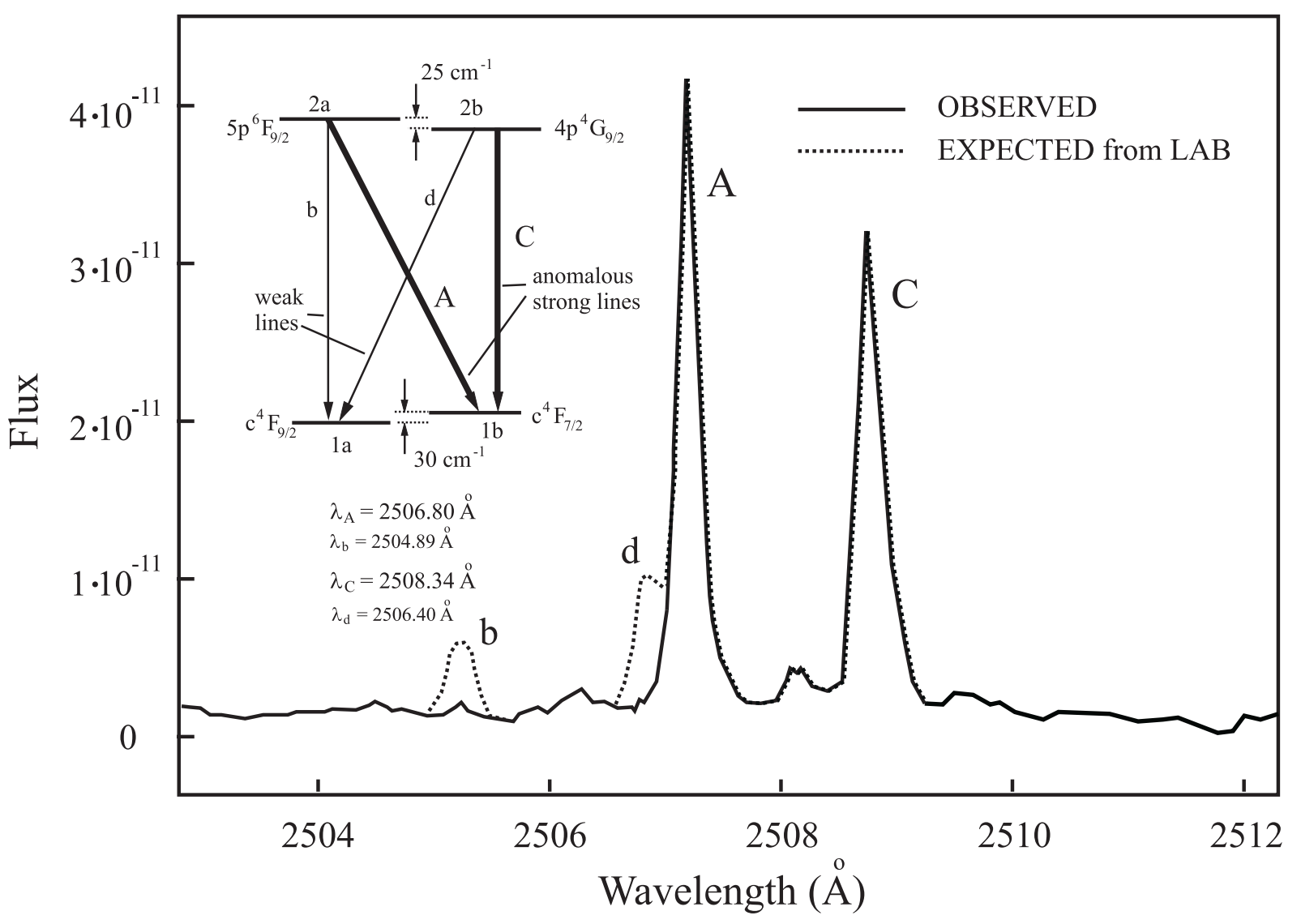

Fig. 4. UV FeII spectral lines (A, C) and their satellites (b, d) observed in blob B (solid line) of $\eta$ Car and relative intensities expected from laboratory data (dashed lines) (according to atomic data in Johansson \& Zethson 1999).

The lower states 1 have long lifetimes with a decay rate of $A_{21} \simeq 0.7 \times 10^{3} \mathrm{~s}^{-1}$ (Kurucz 1988), but the upper states 2 $\left(5 \mathrm{p}^{6} \mathrm{~F}_{9 / 2}\right.$ and $\left.4 \mathrm{p}^{4} \mathrm{G}_{9 / 2}\right)$ have short life times and fast decays with $A_{21} \simeq 10^{8} \mathrm{~s}^{-1}$, typical for allowed transitions. This means that under steady-state conditions (on a time scale of $\geq 10^{-8} \mathrm{~s}$ ) amplification is only possible in the case of fast depletion of the lower states 1 with the rate $W_{\mathrm{dep}}^{1}$ :

$W_{\mathrm{dep}}^{1} \geq A_{21} \simeq 10^{8} \mathrm{~s}^{-1}$.

We can consider the following two very fast depopulation alternatives:

(1) An accidental coincidence of some bright lines with an unknown absorption line from state 2 , and the occurrence of an accidental coincidence of HLy $\alpha$ with the $m \rightarrow 2$ transition in FeII. The probability of an accidental wavelength coincidence for two pairs of lines is extremely low.

(2) Photoionization of FeII in the long-lived state 1 by VUV black body radiation (BBR) with $h \nu>9.94 \mathrm{eV}$ and trapped Ly $\alpha$ emission with $h \nu=10.2 \mathrm{eV}$ generated inside the blob (due to photoionization of HI by absorption of photons with $h \nu \geq 13.6 \mathrm{eV}$ ) with the rate $W_{\mathrm{ph}}^{1 \mathrm{i}}>A_{2 \mathrm{~m}}$ in accordance with the requirement in Eq. (19).
As shown in our preceding work (Johansson \& Letokhov 2001b), $W_{\mathrm{ph}}^{1 \mathrm{i}}$ can exceed the decay rate of the lower level 1 , but it is smaller by several orders of magnitude than the value required by Eq. (19). Nevertheless, this process is potentially important in reducing the accumulation of FeII in the long-lived state 1 and hence the optical density $\tau_{12}$ of the anomalous transition $1 \rightarrow 2$.

\section{The effect of different resonance scattering lengths in optically dense media in the presence of nonresonant absorption}

An essential result of the discussion in (Johansson \& Letokhov 2001b) is the possible evidence for a (in the case of bright UV lines) significant optical density, $\tau_{12}>1$, for UV transitions terminating on long-lived states. A second important feature is the physical difference between the strong and weak pairs of UV lines, namely, that they terminate on different fine-structure levels (!), which we have denoted as levels 1 . Under laboratory conditions (optically thin, non-absorbing media), the intensity ratio 


\section{Normalized Intensities of Spectral Lines}
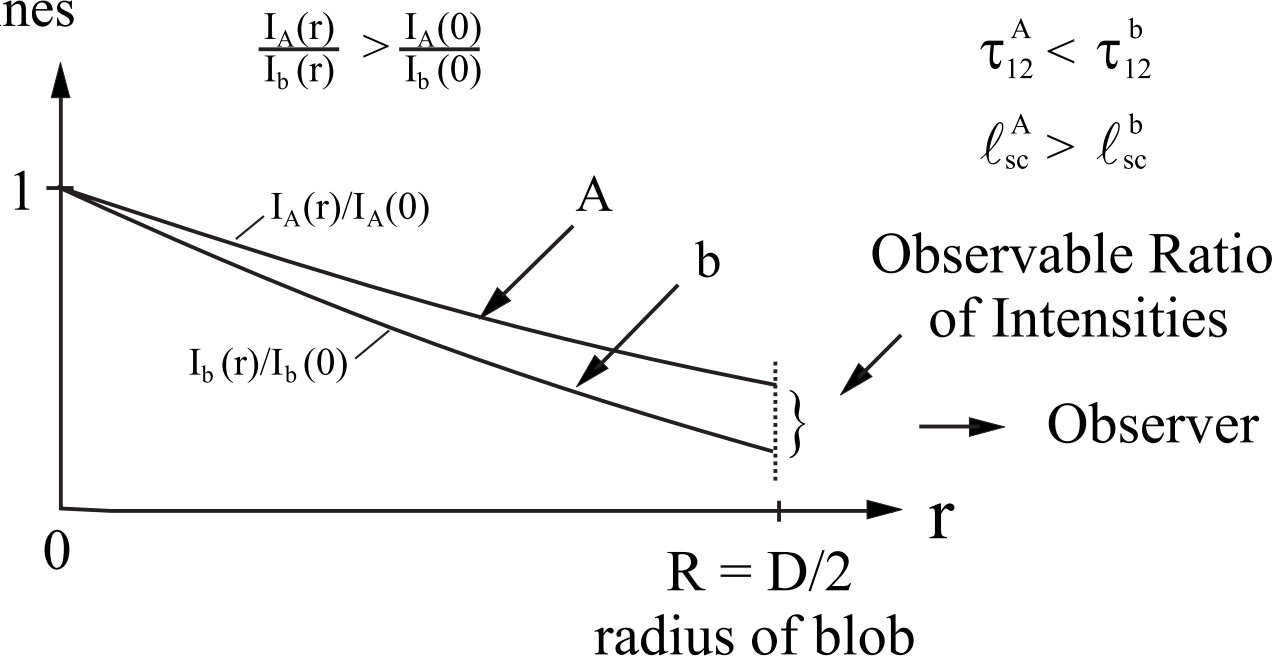

Fig. 5. Qualitative explanation of the intensity variation for the pair of a strong (A) and a weak (b) spectral line due to radiative transfer from the centre $(r=0)$ to the surface $(r=D / 2)$ of the blob. The line intensities are normalized to the laboratory values. The same explanation is also valid for the another pair of strong $(\mathrm{C})$ and weak (d) lines.

between, for example, transition $\mathrm{A}(2 \mathrm{a} \rightarrow 1 \mathrm{~b})$ and transition $\mathrm{b}(2 \mathrm{a} \rightarrow 1 \mathrm{a})$ is:

$\left(I_{\mathrm{A}} / I_{\mathrm{b}}\right)^{\mathrm{Lab} .}=R_{\mathrm{A} / \mathrm{b}}^{\mathrm{Lab}}=\frac{A_{2 \mathrm{a} \rightarrow 1 \mathrm{~b}}}{A_{2 \mathrm{a} \rightarrow 1 \mathrm{a}}} \frac{N_{2 \mathrm{a}}}{N_{2 \mathrm{a}}}=\frac{A_{2 \mathrm{a} \rightarrow 1 \mathrm{~b}}}{A_{2 \mathrm{a} \rightarrow 1 \mathrm{a}}}$

i.e., equal to the ratio between the Einstein coefficients A for the corresponding transitions.

Under blob conditions, we should take into account the possible difference in optical pathway for photons associated with different transitions as a result of a possible difference in the mean resonance scattering lengths in optically thick media $\left(\tau_{12}>1\right)$. The resulting difference in attenuation, $K_{\text {att }}$, in the presence of weak nonresonant (continuous) absorption

$K_{\mathrm{att}}^{\mathrm{A}, \mathrm{b}} \simeq \exp \left(-\kappa_{\mathrm{abs}}<L_{\mathrm{A}, \mathrm{b}}>\right)$,

where $\kappa_{\text {abs }}$ is the nonresonance absorption coefficient $\left(\mathrm{cm}^{-1}\right), L_{\mathrm{A}}$ and $L_{\mathrm{b}}$ are the optical pathways of photons with different resonance scattering coefficients, $\kappa_{\mathrm{sc}}^{\mathrm{A}}$ and $\kappa_{\mathrm{sc}}^{\mathrm{b}}$, or different resonance scattering with mean free paths $\left\langle\ell_{\mathrm{sc}}^{\mathrm{A}}\right\rangle$ and $\left\langle\ell_{\mathrm{sc}}^{\mathrm{b}}\right\rangle$. In the diffusion approximation $\left(<\ell_{\mathrm{sc}}^{\mathrm{A}, \mathrm{b}}>\ll D\right)$

$\left\langle L_{\mathrm{A}, \mathrm{b}}\right\rangle \simeq \frac{D^{2}}{\left\langle\ell_{\mathrm{sc}}^{\mathrm{A}, \mathrm{b}}\right\rangle}=\kappa_{\mathrm{sc}}^{\mathrm{A}, \mathrm{b}} D^{2}$,

where $D$ is the distance from the emitting source to the boundary of the medium (in our case, it is the size of the diameter of the blob), the resonance scattering coefficients are determined by the populations of the lower states $\left(N_{2} \ll N_{1}\right)$, and the corresponding cross-sections or optical densities $\tau_{12}$ of the transitions are given by

$\kappa_{\mathrm{sc}}^{\mathrm{A}}=N_{1 \mathrm{~b}} \sigma_{1 \mathrm{~b}-2 \mathrm{a}}=N_{1 \mathrm{~b}} \sigma_{2 \mathrm{a}-1 \mathrm{~b}} \frac{g_{2 \mathrm{a}}}{g_{1 \mathrm{~b}}} \propto \tau_{1 \mathrm{~b}-2 \mathrm{a}}$ and

$\kappa_{\mathrm{sc}}^{\mathrm{b}}=N_{1 \mathrm{a}} \sigma_{1 \mathrm{a}-2 \mathrm{a}}=N_{1 \mathrm{a}} \sigma_{2 \mathrm{a}-1 \mathrm{a}} \frac{g_{2 \mathrm{a}}}{g_{1 \mathrm{a}}} \propto \tau_{1 \mathrm{a}-2 \mathrm{a}}$.

According to Eqs. (21) and (22), the intensity ratios of the blob lines should be

$$
\left(\frac{I_{\mathrm{A}}}{I_{\mathrm{b}}}\right)^{\text {blob }}=\left(\frac{I_{\mathrm{A}}}{I_{\mathrm{b}}}\right)^{\mathrm{lab}} \mathrm{e}^{-\kappa_{\mathrm{abs}} D^{2}\left(\kappa_{\mathrm{sc}}^{\mathrm{A}}-\kappa_{\mathrm{sc}}^{\mathrm{b}}\right)} \text {. }
$$

Thus, in the presence of nonresonant absorption $\left(\kappa_{\text {abs }} \neq\right.$ 0 ) the difference between the resonance scattering lengths $1 / \kappa_{\mathrm{rs}}^{\mathrm{A}}$ and $1 / \kappa_{\mathrm{sc}}^{\mathrm{b}}$ for different spectral lines will lead to a difference between the blob and laboratory intensity ratios. In Fig. 5 we have plotted the intensities of lines A and $\mathrm{b}$ (normalized to laboratory values) as subject to radiative transfer from the center of the blob $(r=0)$ to its surface $(r=D / 2)$. (Note that the label on the vertical axis in a similar figure in our previous work (Johansson \& Letokhov 2001a) is incorrect.)

The effect of nonresonant absorption of the flux of emission lines having different optical path lengths in a resonance-scattering medium of large optical thickness was considered long ago (Hummer 1968). We have, in essence, rediscovered this effect when trying to find an explanation for the anomalous behavior of the intensities of two pairs of close spectral lines. The qualitative picture above does not take into account the Doppler frequency shift caused by resonance scattering by moving FeII ions. Actually the frequency-shifted photons can escape from the blob at the depth $r_{\text {esc }} \ll D$. This effect will limit the optical pathways $\left\langle L_{\mathrm{A}, \mathrm{b}, \mathrm{C}, \mathrm{d}}\right\rangle$ for trapped spectral lines (Adams 1972; Harrington 1973; Hummer \& Kunasz 1980). Hummer (1968) investigated in detail the special functions arising in radiative transfer due to nonresonance scattering and the numerical solution of the transport equation 
for the case of scattering with complete frequency redistribution. Subject to consideration in Hummer's work (1968) was radiative transfer in a plane-parallel layer with radiation sources both in a line spectrum and in a continuous spectrum. In addition, the source of the line radiation in Hummer's work contained some part due to the absorption of photons as a result of electron collisions with the excited atom. The radiative transfer process in a medium with non-resonant absorption in the case of partial frequency redistribution was investigated both numerically and asymptotically in (Hummer \& Kunasz 1980; Frisch 1980), where effective scaling laws were found for the case of small absorption coefficients and large optical thicknesses of the layer.

In the present work, the transport equations have a somewhat different form, because the only source of radiation in the UV lines is the spontaneous emission of the FeII ions excited by the wide-band Ly $\alpha$ radiation at different wavelength. Furthermore, the optical thickness $\tau_{12}^{0}$ of the $1 \rightarrow 2$ transition, which is responsible for the intense UV radiation, is substantial $\left(\tau_{12}^{0} \gg 1\right)$ (Johansson \& Letokhov 2001b), but not so great that it is necessary to take into account the scattering in the Lorentzian wings, i.e. the parameter $a \tau_{12}^{0}<1$, where the damping parameter $a=\Delta \nu_{\text {rad }} / \Delta \nu_{\text {Dopp }}$ for the FeII lines of interest. And finally, the geometry of the passive region of blob $\mathrm{B}$ exposed to the Ly $\alpha$ radiation is more spherical than plane, even though it is a convenient assumption to simplify the calculations. Thus, for the present problem with the anomalous Fe II lines it would be desirable to calculate the radiative transfer in an optically dense medium featuring nonresonant absorption in a spherical geometry.

\section{The change in intensity of optically thick UV Fell lines in the presence of weak nonresonant absorption (a Spherical Blob)}

Let us consider a gas cloud (or blob) model in the form of a spherical region of radius $R$, which contains FeII ions, which, all over the volume of the region, are subject to homogeneous excitation within the limits of the Doppler profile and, hence, emit spontaneously within the limits of the Doppler line width. The optical thickness of the region, with reference to the line center of the $1 \rightarrow 2$ transition, $\tau_{12}^{0}=\kappa_{\mathrm{sc}} R$ (notation in Johansson \& Letokhov 2001b), will vary over the range $10^{-1} \ldots 10^{2}$, where $\kappa_{\mathrm{sc}}=\sigma_{12} N_{1}$ is the coefficient of resonance scattering per unit length, $\tau_{12}^{0}$ is the radiative transition cross section with reference to the line center, and $N_{1}$ is the concentration of the FeII ions in the state 1 . The coefficient of nonresonant (continuous) absorption per unit length, $\kappa_{\mathrm{abs}}$, will vary relative to $\kappa_{\mathrm{sc}}$ within the limits of $\beta=\kappa_{\mathrm{abs}} / \kappa_{\mathrm{sc}}=0 \ldots 0.1$. Thus, at high $\beta$ and $\kappa_{\mathrm{sc}} R$ values $\left(\kappa_{\mathrm{sc}} R \beta>1\right)$ nonresonant absorption per unit physical size is high, but where $\kappa_{\mathrm{sc}} R \beta \ll 1$ nonresonant absorption can be substantial only for a repeated scattering of radiation, which increases the optical path length.

\subsection{Basic radiation transport equations}

The equation for steady-state radiation intensity $J_{\omega}$ inside a blob at the frequency $\omega$ with a spherical scattering diagram has the form (Sobolev 1949a)

$$
\begin{aligned}
& \left(\boldsymbol{\Omega} \cdot \nabla+\kappa_{\mathrm{sc}}(\omega)+\kappa_{\mathrm{abs}}\right) J_{\omega}(\boldsymbol{r}, \boldsymbol{\Omega})= \\
& \quad \frac{1}{4 \pi} \int \mathrm{d} \omega^{\prime} p\left(\omega, \omega^{\prime}\right) \kappa_{\mathrm{sc}}\left(\omega^{\prime}\right) \int J_{\omega^{\prime}}(\boldsymbol{r}, \boldsymbol{\Omega}) \mathrm{d} \boldsymbol{\Omega}+I_{0}(\omega)
\end{aligned}
$$

where the spontaneous emission intensity of FeII at level 2 is described by the term

$I_{0}(\omega)=\frac{A}{4 \pi c} \kappa_{\mathrm{sc}}(\omega) W_{0}, \quad A=\frac{1}{\int \kappa_{\mathrm{sc}}(\omega) \mathrm{d} \omega}$

and the normalization corresponds to the radiation density (in photons $\left./ \mathrm{cm}^{3}\right)$. In this equation, $J_{\omega}(\boldsymbol{r}, \boldsymbol{\Omega}) \mathrm{d} \omega \mathrm{d} \boldsymbol{r} \mathrm{d} \boldsymbol{\Omega}$ is the number of photons contained in the volume $\mathrm{d} \boldsymbol{r}$, having their frequencies in the interval $\mathrm{d} \omega$ and moving within the limits of the solid angle $\mathrm{d} \boldsymbol{\Omega}$. The factor $p\left(\omega, \omega^{\prime}\right)$ is the probability that a photon of frequency $\omega$ will be emitted after the atom has interacted with a photon of frequency $\omega^{\prime}, \Omega$ is the unit vector of the photon motion direction, and $\kappa_{\mathrm{sc}}(\omega)$ and $\kappa_{\text {abs }}$ are the coefficients of resonance scattering and nonresonant absorption per unit length at the frequency $\omega$. In the subsequent discussion we assume that the frequency dependence of the scattering coefficient is due to the Maxwellian atomic velocity distribution and has the form

$\kappa_{\mathrm{sc}}(\omega)=\kappa_{\mathrm{sc}} \exp \left(-\frac{z^{2}}{2}\right), \quad z=\frac{\left(\omega-\omega_{0}\right)}{\Delta \omega_{\mathrm{D}}} \sqrt{2}$.

Here we use the Doppler width definition, which is adopted in the astrophysical literature (Mihalas 1978). This definition differs only by a factor of $(4 \ln 2)^{-1 / 2}$ from the definition used in optical spectroscopy (Svelto 1989).

The transport Eq. (26) differs from the commonly used one in two aspects. Firstly, intending to describe radiation in spherical blobs, we consider a three-dimensional, spherically symmetric problem, whereas it is usually a twodimensional, plane problem that is subject to consideration. Secondly, we assume that the frequency dependence of the radiation source, $I_{0}(\omega)$, is defined by the Doppler profile.

Since the optical thickness we consider for the $1 \rightarrow 2$ UV transition in FeII is not very large $\left(\tau_{12}^{0} a<1\right)$, scattering takes place inside the Doppler core (Mihalas 1978). In that case, photon scattering is completely noncoherent, i.e., the scattering probability $p\left(\omega, \omega^{\prime}\right)$ has the following form:

$p\left(\omega, \omega^{\prime}\right)=A \kappa_{\mathrm{sc}}(\omega)$.

In this approximation, Eq. (7) adopts the form

$$
\begin{aligned}
(\boldsymbol{\Omega} \cdot \nabla+ & \left.\kappa_{\mathrm{sc}}(\omega)+\kappa_{\mathrm{abs}}\right) J_{\omega}(\boldsymbol{r}, \boldsymbol{\Omega})=\frac{A}{4 \pi} \kappa_{\mathrm{sc}}(\omega) \\
& \times\left(\int \mathrm{d} \omega^{\prime} \kappa_{\mathrm{sc}}\left(\omega^{\prime}\right) \int J_{\omega^{\prime}}(\boldsymbol{r}, \boldsymbol{\Omega}) \mathrm{d} \boldsymbol{\Omega}+\frac{1}{c} W_{0}\right) .
\end{aligned}
$$


Equation (30) describes the transfer of UV radiation inside the blob. To fully formulate the problem, it is necessary to specify the boundary conditions at the edge of the blob, which assumes no incoming flux into the blob. However, it seems to be more convenient to formulate the problem in another way, where we assume that radiation propagates in an infinite space, but scattering occurs only at $r<$ $R$. One can easily see that also in this case there is no incoming flux on the surface of the blob, and by virtue of that fact, the solutions of the different approaches to the same problem coincide inside the blob. Thus, we have to solve the equation

$$
\begin{aligned}
& \left(\boldsymbol{\Omega} \cdot \nabla+\kappa_{\mathrm{sc}}(\omega)+\kappa_{\mathrm{abs}}\right) J_{\omega}(\boldsymbol{r}, \boldsymbol{\Omega})= \\
& \left\{\begin{array}{c}
\frac{A}{4 \pi} \kappa_{\mathrm{sc}}(\omega)\left(\int \mathrm{d} \omega^{\prime} \kappa_{\mathrm{sc}}\left(\omega^{\prime}\right) \int J_{\omega^{\prime}}(\boldsymbol{r}, \boldsymbol{\Omega}) \mathrm{d} \boldsymbol{\Omega}+\frac{1}{c} W_{0}\right), r<R \\
0,
\end{array} \quad r>R .\right.
\end{aligned}
$$

If we do not consider the distribution of photons over the propagation directions, we then can exclude from Eq. (31) the dependence on the variable $\boldsymbol{\Omega}$. This can be achieved by taking the Fourier transform in terms of spatial coordinates and then averaging over propagation directions. As a result, we obtain the following integral equation for the spectral density of photons in space, $\bar{J}(\boldsymbol{r}, \omega)=\int \mathrm{d} \boldsymbol{\Omega} J_{\omega}(\boldsymbol{r}, \boldsymbol{\Omega}):$

$$
\begin{aligned}
\bar{J}(\boldsymbol{r}, \omega)=\frac{A}{4 \pi} & \kappa_{\mathrm{sc}}(\omega) \int_{\left|\boldsymbol{r}^{\prime}\right|<R} \frac{\mathrm{d}^{3} \boldsymbol{r}}{\left|\boldsymbol{r}-\boldsymbol{r}^{\prime}\right|^{2}} \mathrm{e}^{-\left(\kappa_{\mathrm{sc}}(\omega)+\kappa_{\mathrm{abs}}\right)\left|\boldsymbol{r}-\boldsymbol{r}^{\prime}\right|} \\
& \times\left\{\int \mathrm{d} \omega^{\prime} \kappa_{\mathrm{sc}}\left(\omega^{\prime}\right) \bar{J}\left(\boldsymbol{r}^{\prime}, \omega^{\prime}\right)+\frac{1}{c} W_{0}\right\} \cdot
\end{aligned}
$$

As already noted, this equation correctly describes the true photon density inside the blob only. In the case of spherical symmetry of the problem, Eq. (32) may be reduced to the form (Sobolev 1949b):

$$
\begin{aligned}
r \bar{J}(r, \omega)= & \frac{A \kappa_{\mathrm{sc}}(\omega)}{2} \int_{-R}^{R} r^{\prime} E_{1}\left(\left|r-r^{\prime}\right|\left(\kappa_{\mathrm{sc}}(\omega)+\kappa_{\mathrm{abs}}\right)\right) \\
& \times\left(\int \mathrm{d} \omega^{\prime} \kappa_{\mathrm{sc}}\left(\omega^{\prime}\right) \bar{J}\left(r^{\prime}, \omega^{\prime}\right)+\frac{1}{c} W_{0}\right) \mathrm{d} r^{\prime}
\end{aligned}
$$

where $E_{1}$ is the integral exponential function (Abramovitz 1964).

For the total photon density in space, $J^{*}(r)=$ $\int \mathrm{d} \omega^{\prime} \kappa_{\text {sc }}\left(\omega^{\prime}\right) \bar{J}\left(r, \omega^{\prime}\right)$, we have from Eq. (33)

$$
\begin{aligned}
r J^{*}(r) & =\frac{A}{2} \int_{-R}^{R} r^{\prime}\left(J^{*}\left(r^{\prime}\right)+\frac{1}{c} W_{0}\right) \mathrm{d} r^{\prime} \\
& \times \int \mathrm{d} \omega \kappa_{\mathrm{sc}}^{2}(\omega) E_{1}\left(\left|r-r^{\prime}\right|\left(\kappa_{\mathrm{sc}}(\omega)+\kappa_{\mathrm{abs}}\right)\right) .
\end{aligned}
$$

Introducing the effective photon density (resonance-line source function)

$Q(r)=r\left(J^{*}(r)+\frac{1}{c} W_{0}\right)$ we may represent the final integral equation in the form

$$
Q(r)=\int_{-R}^{R} Q\left(r^{\prime}\right) \mathrm{d} r^{\prime} K\left(\left|r-r^{\prime}\right|\right)+r \frac{W_{0}}{c}
$$

where

$$
K\left(\left|r-r^{\prime}\right|\right)=\frac{A}{2} \int \mathrm{d} \omega \kappa_{\mathrm{sc}}^{2}(\omega) E_{1}\left(\left|r-r^{\prime}\right|\left(\kappa_{\mathrm{sc}}(\omega)+\kappa_{\mathrm{abs}}\right)\right)
$$

and $-R<r, r^{\prime}<R$.

Given the solution of Eq. (37), we can find the spectral photon density $\bar{J}(r, \omega)$ and the total photon density $P(r)$ at any point inside the blob:

$$
\begin{aligned}
r \bar{J}(r, \omega) & =\frac{A \kappa_{\mathrm{sc}}(\omega)}{2} \\
& \times \int_{-R}^{R} E_{1}\left(\left|r-r^{\prime}\right|\left(\kappa_{\mathrm{sc}}(\omega)+\kappa_{\mathrm{abs}}\right)\right) Q\left(r^{\prime}\right) \mathrm{d} r^{\prime} \\
P(r)= & \int \mathrm{d} \omega \bar{J}(r, \omega)=\frac{A}{2 r} \int_{-R}^{R} Q\left(r^{\prime}\right) \mathrm{d} r^{\prime} \\
& \times \int \mathrm{d} \omega^{\prime} \kappa_{\mathrm{sc}}\left(\omega^{\prime}\right) E_{1}\left(\left|r-r^{\prime}\right|\left(\kappa_{\mathrm{sc}}\left(\omega^{\prime}\right)+\kappa_{\mathrm{abs}}\right)\right) .
\end{aligned}
$$

At the edge of the blob $(r=R)$ we have

$$
\begin{aligned}
& \bar{J}(R, \omega)=\frac{A \kappa_{\mathrm{sc}}(\omega)}{2 R} \\
& \times \int_{-R}^{R} E_{1}\left(\left|R-r^{\prime}\right|\left(\kappa_{\mathrm{sc}}(\omega)+\kappa_{\mathrm{abs}}\right)\right) Q\left(r^{\prime}\right) \mathrm{d} r^{\prime} \\
& P(R)=\int \mathrm{d} \omega \bar{J}(R, \omega)=\frac{A}{2 R} \int_{-R}^{R} Q\left(r^{\prime}\right) \mathrm{d} r^{\prime} \\
& \times \int \mathrm{d} \omega^{\prime} \kappa_{\mathrm{sc}}\left(\omega^{\prime}\right) E_{1}\left(\left|R-r^{\prime}\right|\left(\kappa_{\mathrm{sc}}\left(\omega^{\prime}\right)+\kappa_{\mathrm{abs}}\right)\right) .
\end{aligned}
$$

\subsection{Numerical solution of the integral equation}

The integral equation (Eq. (26)) can be solved either numerically or by the approximate analytical methods (Sobolev 1949a; Ivanov 1973). In this work, we solve this integral equation numerically, as we are interested in the radiation characteristics at a wide variety of values of the scattering, absorption, etc. parameters, and analytical solutions are usually applicable only within limited parameter regions. The dimensionless form of Eq. (36) is as follows:

$$
\begin{aligned}
\tilde{Q}(x)= & \frac{\Lambda}{2 \sqrt{2 \pi}} \int_{-1}^{1} \tilde{Q}\left(x^{\prime}\right) \mathrm{d} x^{\prime} \\
& \times \int_{-\infty}^{1} \mathrm{~d} \xi \alpha^{2}(\xi) E_{1}\left(\left|x-x^{\prime}\right| \tilde{\Lambda}(\xi)\right)+x,
\end{aligned}
$$$$
\text { where }-1<x, x^{\prime}<1 \text {. }
$$ 
When obtaining Eqs. (36) to (42), we have introduced the following notations:

$$
\begin{aligned}
& \tilde{Q}(x)=\frac{c Q(r)}{R W_{0}} ; x=\frac{r}{R} ; \Lambda=\kappa_{\mathrm{sc}} R \\
& \tilde{\Lambda}(\xi)=\Lambda[\alpha(\xi)+\beta], \alpha(\xi)=\exp \left(-\frac{\xi^{2}}{2}\right) .
\end{aligned}
$$

The integral transport Eq. (42) is often solved by approximating the kernel with a set of exponents (Avrett \& Hummer 1965). This approach, however, imposes restrictions upon the form of the right-hand side of Eq. (26) and fails to describe the singular character of the kernel adequately enough.

In the present work, we use the Galerkin method (Kalitkin 1978) and expand the solution $Q(x)$ of Eq. (42) into a trigonometric series:

$Q(x)=\alpha x+\sum_{m=1}^{\infty} y_{m} \sin \pi m x$

The choice of expansion (28) is due to the odd character of $Q(x)$ and the fact that $Q( \pm 1) \neq 0$ at the boundaries of the region. Substituting expression (28) into (21), we get an infinite set of linear equations for $y_{n}$ :

$y_{m}-\sum_{n} A_{m n} y_{n}=\alpha b_{m}-\frac{2(-1)^{n}}{\pi n}(1-\alpha)$

where

$$
\begin{aligned}
& A_{m n}=\frac{\Lambda}{\sqrt{2 \pi}} \int_{-\infty}^{\infty} \mathrm{d} \xi \alpha^{2}(\xi) G_{m n}(\xi) \\
& b_{m}=\frac{\Lambda}{\sqrt{2 \pi}} \int_{-\infty}^{\infty} \mathrm{d} \xi \alpha^{2}(\xi) H_{m}(\xi)
\end{aligned}
$$

and

$$
G_{m n}(\xi)=\frac{1}{2} \int_{-1}^{1} \mathrm{~d} x \mathrm{~d} x^{\prime} \sin \pi m x \sin \pi n x^{\prime} E_{1}\left(\left|x-x^{\prime}\right| \tilde{\Lambda}(\xi)\right)
$$

$$
H_{m}(\xi)=\frac{1}{2} \int_{-1}^{1} \mathrm{~d} x \mathrm{~d} x^{\prime} x^{\prime} \sin \pi m x E_{1}\left(\left|x-x^{\prime}\right| \tilde{\Lambda}(\xi)\right)
$$

The integrals $G_{m n}$ and $H_{m}$ can be expressed in terms of integral exponential functions of complex variable:

$$
\begin{aligned}
G_{m n}= & \frac{(-1)^{m+n} m}{\pi^{2} n\left(m^{2}-n^{2}\right)}\left\{\frac{1}{2} \ln \left(1+\frac{\pi^{2} n^{2}}{\tilde{\Lambda}^{2}(\xi)}\right)-E_{1}(2 \tilde{\Lambda}(\xi))\right. \\
& \left.+\Re\left(E_{1}(2 \tilde{\Lambda}(\xi)+2 \pi n i)\right)\right\}+(m \Leftrightarrow n)
\end{aligned}
$$

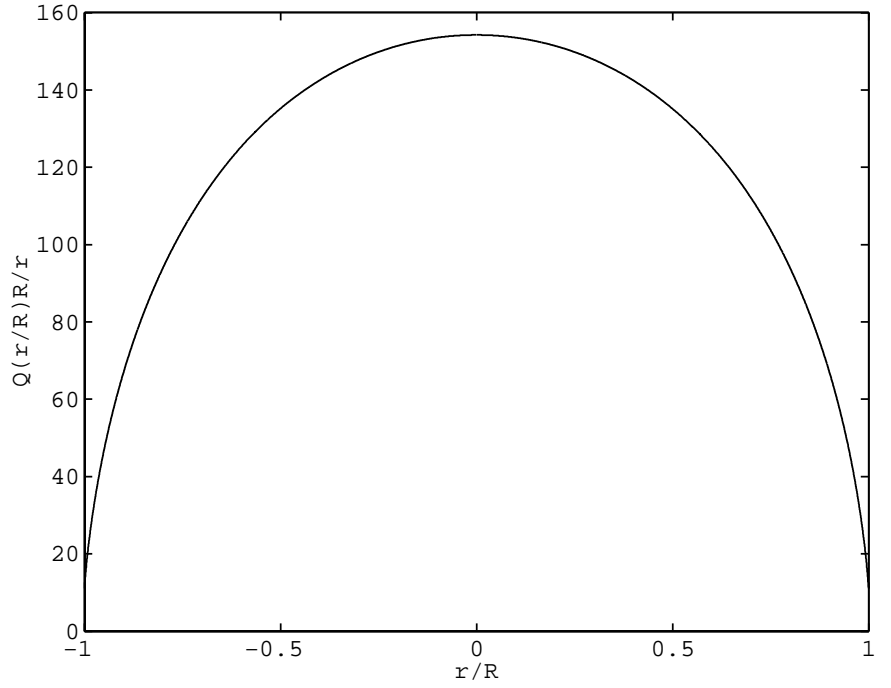

Fig. 6. Source function $Q(x)$ at $\tau_{12}=\kappa_{\mathrm{sc}} R=100$, and $\beta=$ $\kappa_{\mathrm{abs}} / \kappa_{\mathrm{sc}}=0.001$.

$$
\begin{aligned}
G_{n n} & =\frac{1}{2 \pi^{2} n^{2}}\left\{\ln \left(1+\frac{\pi^{2} n^{2}}{\tilde{\Lambda}^{2}(\xi)}\right)-2 \pi n \arctan \frac{\tilde{\Lambda}(\xi)}{\pi n}\right. \\
& \left.+\pi^{2} n-\frac{\pi^{2} n^{2}}{\tilde{\Lambda}^{2}(\xi)+\pi^{2} n^{2}}\left(1-\mathrm{e}^{-2 \tilde{\Lambda}(\xi)}\right)\right\}-\frac{1}{\pi^{2} n^{2}} \\
& \times\left\{E_{1}(2 \tilde{\Lambda}(\xi))-\Re\left((1-\pi n i) E_{1}(2 \tilde{\Lambda}(\xi)+2 \pi n i)\right)\right\}
\end{aligned}
$$

$$
\begin{aligned}
H_{m} & =\frac{(-1)^{n}}{(\pi n)^{3}}\left\{-\frac{1}{2} \ln \left(1+\frac{\pi^{2} n^{2}}{\tilde{\Lambda}^{2}(\xi)}\right)+\pi n \arctan \frac{\tilde{\Lambda}(\xi)}{\pi n}\right. \\
& \left.-\frac{\pi^{2} n}{2}+\frac{\pi^{2} n^{2}}{2 \tilde{\Lambda}^{2}(\xi)}\left(1-\mathrm{e}^{-2 \tilde{\Lambda}(\xi)}\right)-\frac{\pi^{2} n^{2}}{\tilde{\Lambda}(\xi)}\right\}+\frac{(-1)^{n}}{(\pi n)^{3}} \\
& \times\left\{E_{1}(2 \tilde{\Lambda}(\xi))-\Re\left((1-\pi n i) E_{1}(2 \tilde{\Lambda}(\xi)+2 \pi n i)\right)\right\} .
\end{aligned}
$$

The analytical calculation of integrals (50)-(52) in terms of coordinates allows one to avoid the difficulties associated with the singular character of the kernel. The integrals in terms of the dimensionless frequency $\xi$ are computed numerically. The infinite system (45) can be reduced to a finite-dimensional one by simply eliminating the higher harmonics. In our numerical computations, we included up to 700 harmonics. In doing so, we determined the parameter $\alpha$ in this system so as to minimize oscillations at the point $x=0$. The absence of oscillations corresponded to the optimum choice of $\alpha=\tilde{Q}(1)$. With the parameter $\alpha$ determined in this way, the Fourier series was summed by the Fejer method (Courant \& Hilbert 1962). As a result, we obtained a smooth solution of Eq. (42). Figure 6 shows the solution of Eq. (42) at typical parameter values $(\Lambda=100, \beta=0.001)$.

With integral Eq. (42) solved, the spectral radiation density and photon density were found by direct numerical integration in accordance with expressions (38)-(41). 


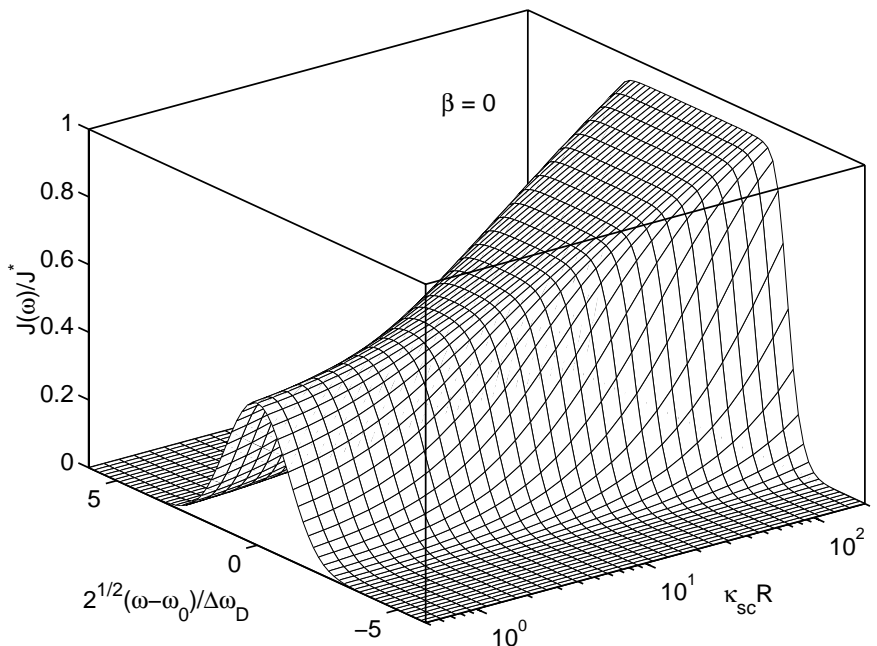

Fig. 7. Spectral radiation density at the center of the blob, $\bar{J}(r=0, \omega)$, as a function of the optical density in the absence of nonresonance absorption $\left(\tau_{12}=\kappa_{\mathrm{sc}} R=0.5 \ldots 200, \beta=\right.$ $\left.\kappa_{\mathrm{abs}} / \kappa_{\mathrm{sc}}=0.000\right)$, normalized to the spectral density value at the center of the blob in the absence of scattering.

To answer the question about the effect of continuous absorption on the intensity of the spectral line observed, it is sufficient to calculate the dependence of the spectrumintegrated radiation intensity at the boundary of the spherical region on the parameters $\kappa_{\mathrm{sc}} R$ and $\beta=\kappa_{\mathrm{abs}} / \kappa_{\mathrm{sc}}$. We will, however, present a much greater mass of numerical data obtained, for they may also prove to be of interest in understanding the origin and evolution of spectral lines inside the blob.

Figures 7 and 8 present the dependences of the spectral radiation density at the center of the spherical blob on the transition frequency and optical density $\tau_{12}=\kappa_{\mathrm{sc}} R$ at various values of the nonresonance absorption $\beta=\kappa_{\mathrm{abs}} / \kappa_{\mathrm{sc}}$. It can be seen from these figures that when absorption is absent $(\beta=0)$ (Fig. 7$)$, the line both broadens monotonically and increases in intensity as the optical density grows higher. When absorption is nonzero (Fig. 8), the line broadens with increasing optical density, but its intensity reaches a maximum and then decreases. As absorption grows higher (Fig. 8), the line intensity already starts diminishing at low optical density values. Such a behavior of both the broadening of the line and the reduction of its intensity with increasing absorption is quite understandable: the line broadening is associated with non-coherent scattering in each scattering event, whereas the reduction of the line intensity at the center of the blob is due to the fact that the photons, which formerly came to the center of the blob from its intermediate layers, can no longer reach the center because of absorption.

Figures 9 and 10 present the dependences of the spectral radiation density at the edge of the blob on the frequency $\omega$ and optical density $\tau_{12}$ at various nonresonance absorption cross section values. It can be seen from these figures that at low absorption values and sufficiently high $\tau_{12}$ values there is the formation of the well-known doublet line structure (Figs. 9, 10a), which vanishes as absorption grows higher (Figs. 10b, c, and d). This is explained quite naturally: only those photons can reach the boundary of the blob which come from inside the thin layer next to its surface, wherein the number of scattering events is small and so is, accordingly, the spectral diffusion effect.

Figure 11 illustrates the dependence of the total photon density (normalized to the photon density in the absence of scattering) at the center of the blob on the scattering cross section and blob radius at various absorption coefficients. It can be seen that even here, as in the case of spectral radiation density, the photon density in the absence of absorption increases in a monotonic fashion as a result of the diffusive confinement effect. At nonzero absorption values the photon density first grows slightly higher and then decreases substantially.

A similar situation is also obtained for the photon density on the surface of the blob, which governs the line intensity observed (Fig. 12). In this case, however, even in the absence of absorption, the photon density slowly tends to 1 , i.e., the photon density on the surface of the major blob in the absence of scattering, following a slight increase. The most important feature of Fig. 12 is the fact that the photon density rapidly drops with increasing absorption coefficient as a result of the optical path length growing longer in the absorbing medium.

\section{Applications of anomalous UV Fell lines in $\eta$ Carinae}

From the qualitative standpoint, the main result of this computer consideration of Eqs. (43), (46), and (47), which are presented in Fig. 12, is as follows: the change of the attenuation coefficients of two lines is inversely proportional to the resonance scattering coefficients (see the asymptotes for $\kappa_{\mathrm{sc}} R \ll 1$ in Fig. 12). This asymptotic behaviour can be approximated by the simple expression giving the intensity ratio between the blob lines $\mathrm{A}$ and $\mathrm{b}$ on the surface of the blob:

$$
\left(\frac{I_{\mathrm{A}}}{I_{\mathrm{b}}}\right) \sim\left(\frac{\kappa_{\mathrm{sc}}^{\mathrm{b}}}{\kappa_{\mathrm{sc}}^{\mathrm{A}}}\right) .
$$

Using a more detailed notation for the anomalous spectral lines and the corresponding quantum transitions and energy levels (Fig. 13), one can estimate the ratio between the resonance scattering coefficients:

$$
\begin{aligned}
\frac{\kappa_{\mathrm{sc}}^{\mathrm{A}}}{\kappa_{\mathrm{sc}}^{\mathrm{b}}} & =\frac{\tau_{1 \mathrm{~b}-2 \mathrm{a}}}{\tau_{1 \mathrm{a}-2 \mathrm{a}}}=\frac{N_{1 \mathrm{a}}}{N_{1 \mathrm{~b}}} \frac{\sigma_{1 \mathrm{a}-2 \mathrm{a}}}{\sigma_{1 \mathrm{~b}-2 \mathrm{a}}} \\
& =\left(\frac{N_{1 \mathrm{~b}}}{N_{1 \mathrm{a}}}\right)\left(\frac{A_{2 \mathrm{a}-1 \mathrm{~b}}}{A_{2 \mathrm{a}-1 \mathrm{a}}}\right)\left(\frac{g_{1 \mathrm{a}} / g_{2 \mathrm{a}}}{g_{1 \mathrm{~b}} / g_{2 \mathrm{a}}}\right) \\
& =\left(\frac{T_{1 \mathrm{a}}}{T_{1 \mathrm{~b}}}\right)\left(\frac{A_{2 \mathrm{a}-1 \mathrm{a}}}{A_{2 \mathrm{a}-1 \mathrm{~b}}}\right)\left(\frac{g_{1 \mathrm{a}}}{g_{1 \mathrm{~b}}}\right)
\end{aligned}
$$



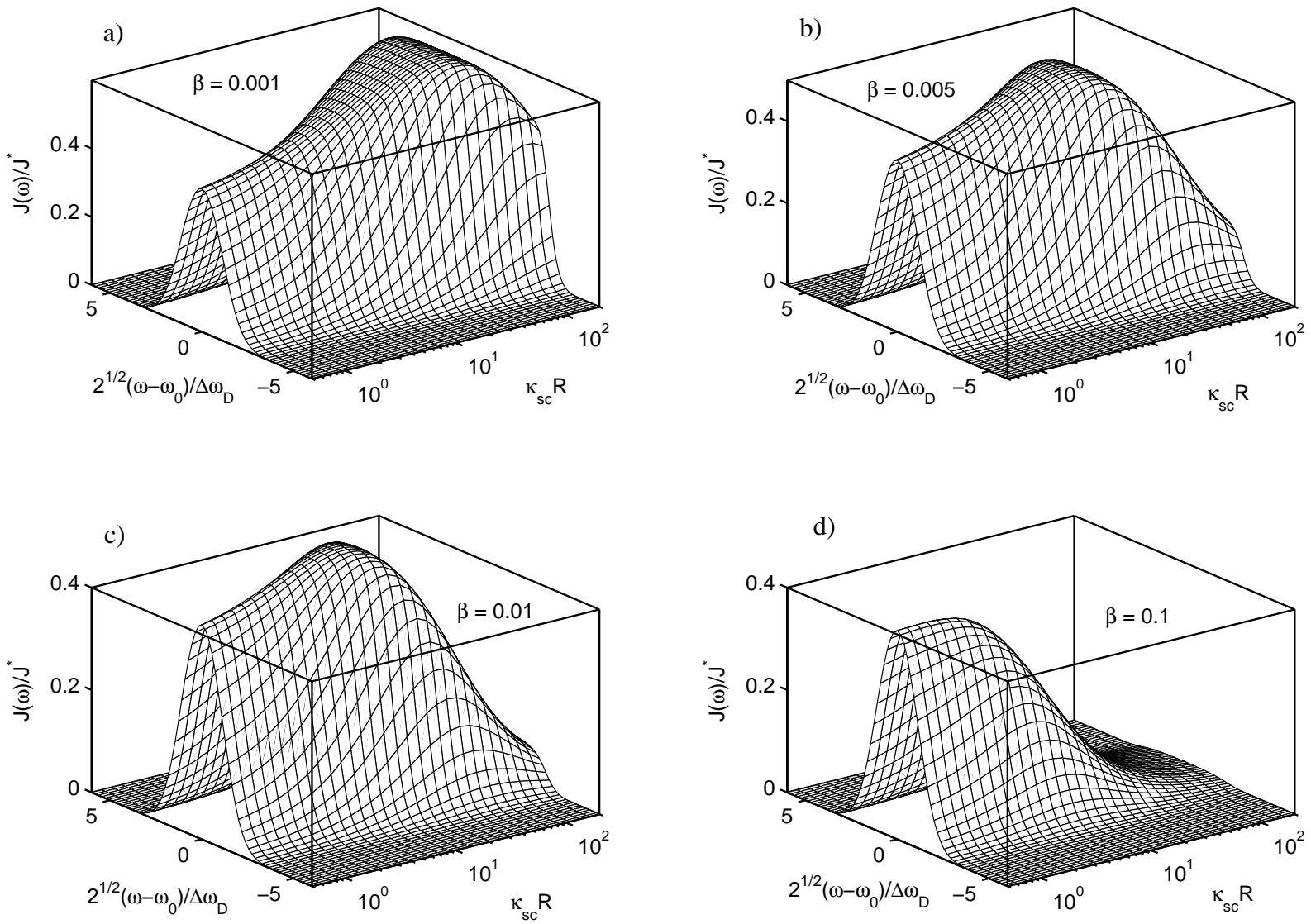

Fig. 8. Spectral radiation density at the center of nebulae, $\bar{J}(r=0, \omega)$, as a function of the optical density $\tau_{12}\left(\tau_{12}=\kappa_{\mathrm{sc}} R=\right.$ $0.5 \ldots 200)$ at various nonresonance absorption coefficients $\left(\beta=\kappa_{\mathrm{abs}} / \kappa_{\mathrm{sc}}\right)$, normalized to the spectral density value at the center of the nebula in the absence of scattering: a) $\beta=0.001$; b) $\beta=0.005$; c) $\beta=0.01$; d) $\beta=0.1$.

Using the asymptotic ratio (53), we can obtain the following expression for the blob and laboratory line intensity ratios:

$$
\left(\frac{I_{\mathrm{A}}}{I_{\mathrm{b}}}\right)^{\mathrm{blob}} \simeq\left(\frac{g_{1 \mathrm{a}}}{g_{1 \mathrm{~b}}}\right)\left(\frac{T_{1 \mathrm{a}}}{T_{1 \mathrm{~b}}}\right) /\left(\frac{I_{\mathrm{A}}}{I_{\mathrm{b}}}\right)^{\text {Lab. }} .
$$

A similar expression can also be obtained for the $(\mathrm{C}, \mathrm{d})$ pair of UV FeII lines:

$$
\left(\frac{I_{\mathrm{C}}}{I_{\mathrm{d}}}\right)^{\text {blob }} \simeq\left(\frac{g_{1 \mathrm{a}}}{g_{1 \mathrm{~b}}}\right)\left(\frac{T_{1 \mathrm{a}}}{T_{1 \mathrm{~b}}}\right) /\left(\frac{I_{\mathrm{C}}}{I_{\mathrm{d}}}\right)^{\text {Lab. }} .
$$

The model considered above for the difference in attenuation between spectral lines in resonance-scattering and nonresonance-absorbing media requires that the difference between the lifetimes $T_{1 \mathrm{a}}$ and $T_{1 \mathrm{~b}}$ of the low-lying levels (1a) and (1b) (or $c^{4} \mathrm{~F}_{9 / 2}$ and $c^{4} \mathrm{~F}_{7 / 2}$ according to the present identification of FeII levels) should be substantial:

$T_{1 \mathrm{a}} \gg T_{1 \mathrm{~b}}$.

It is only in this case that the weak (in lab.) lines are becoming more strongly attenuated in the blob than their strong counterparts. This requirement contradicts the existing data on the lifetimes of the lower levels: $T_{1 \mathrm{a}}=$ $1.35 \mathrm{~ms}, T_{1 \mathrm{~b}}=1.6 \mathrm{~ms}$ (Kurucz 1988).

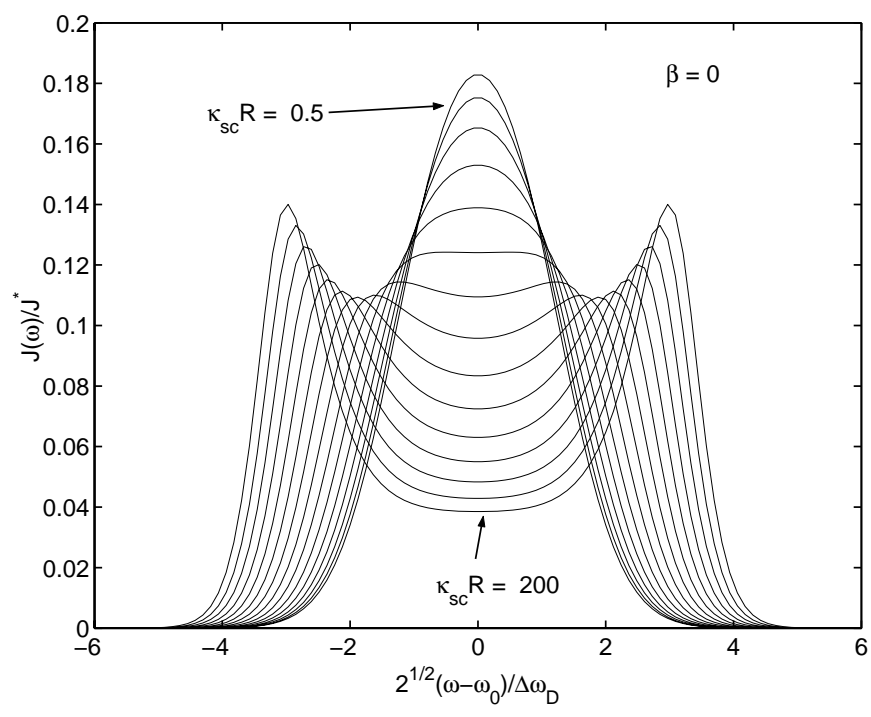

Fig. 9. Spectral radiation density at the edge of the nebula, $\bar{J}(r=R, \omega)$, as a function of the optical density $\tau_{12}=\kappa_{\mathrm{sc}} R$ in the absence of nonresonance absorption $(\beta=0)$, normalized to the spectral density value at the edge of the nebula in the absence of scattering.

To fulfill the requirement, we should consider the possibility of the photoionization depopulation of the lower 

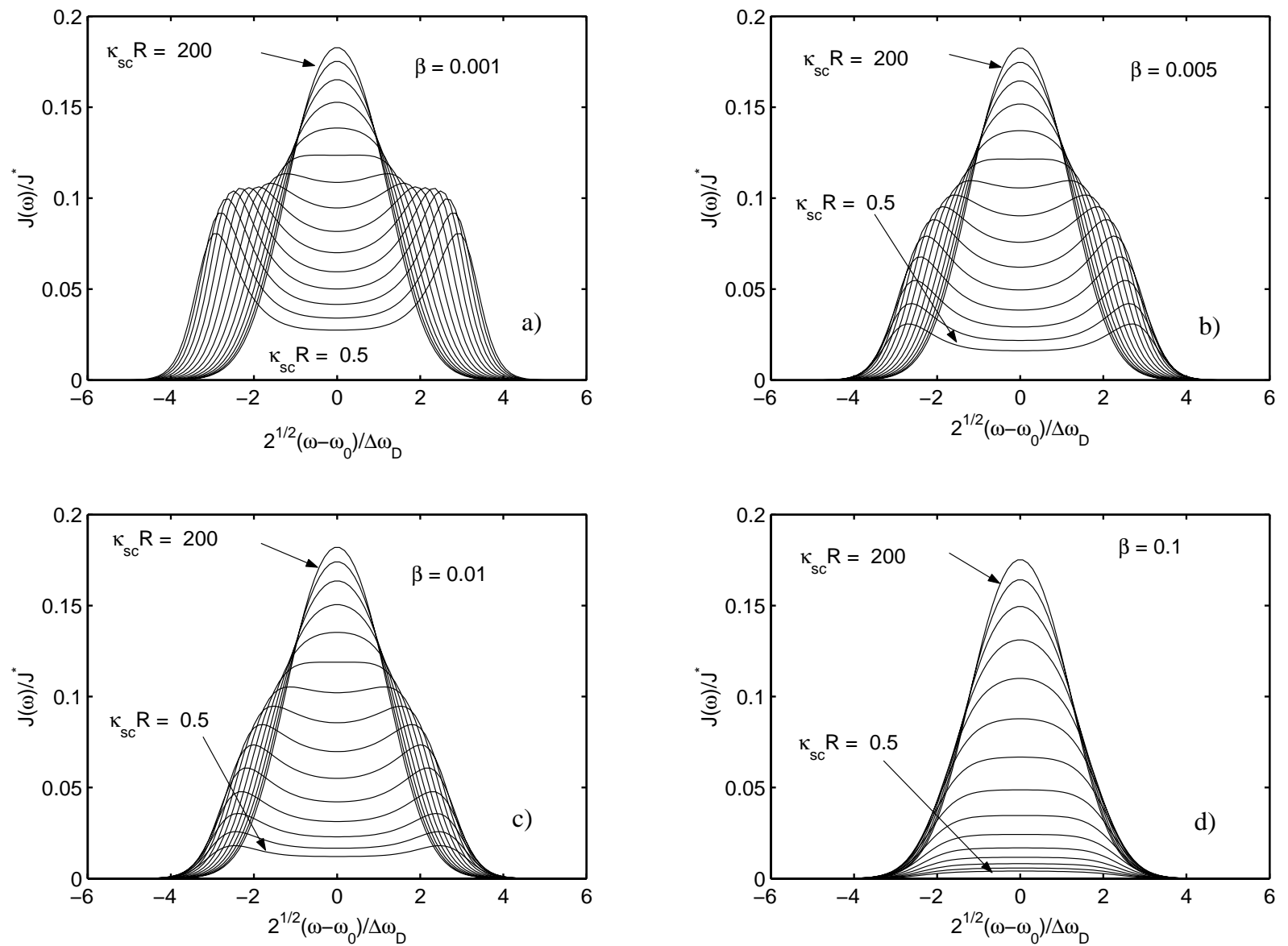

Fig. 10. Spectral radiation density at the edge of the nebula, $\bar{J}(r=R, \omega)$, as a function of the optical density $\tau_{12}=\kappa_{\mathrm{sc}} R=$ $0.5 \ldots 200$ at various nonresonance absorption cross sections, normalized to the spectral density value at the center of the nebula in the absence of scattering: a) $\beta=0.001$; b) $\beta=0.005$; c) $\beta=0.01$; d) $\beta=0.1$.

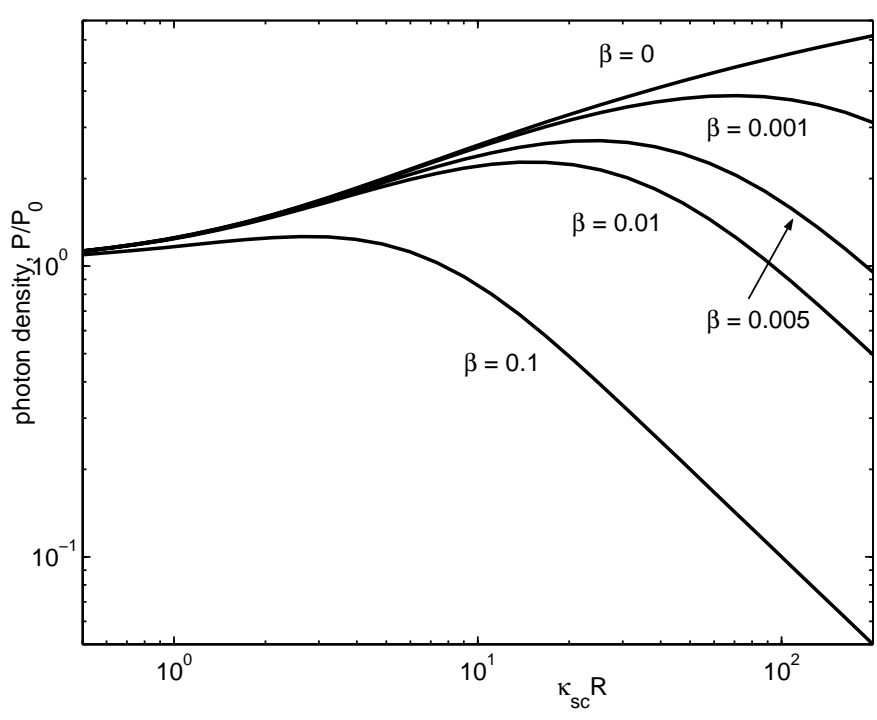

Fig. 11. Total photon density at the center of the nebula as a function of the optical density $\tau_{12}$ at various continuous absorption parameter values, normalized to the total photon density value in the absence of scattering.

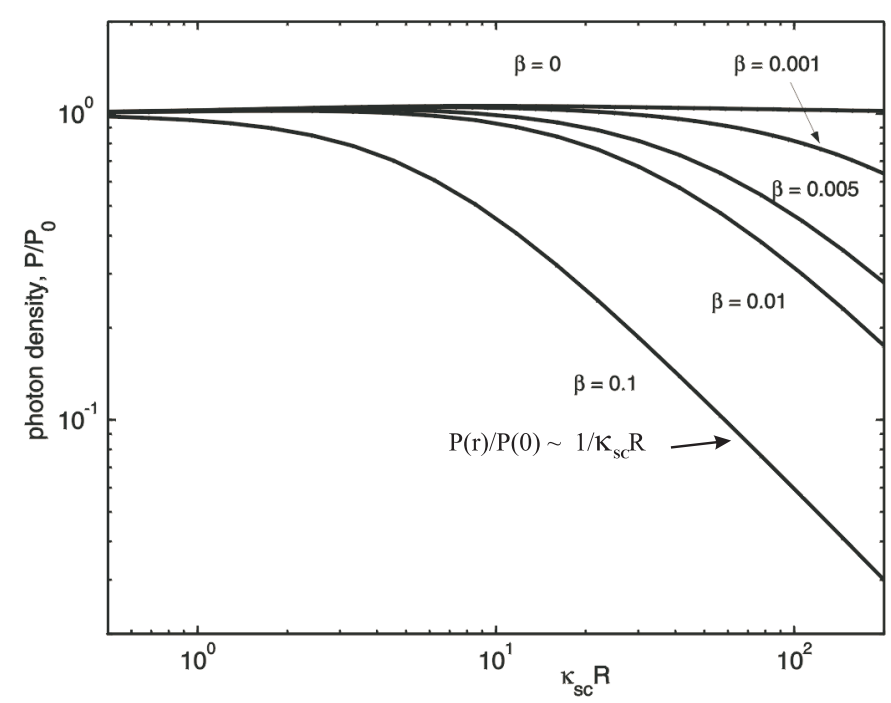

Fig. 12. Total photon density at the edge of the nebula as a function of the optical density $\tau_{12}$ at various continuous absorption parameter values, normalized to the total photon density value in the absence of scattering. 


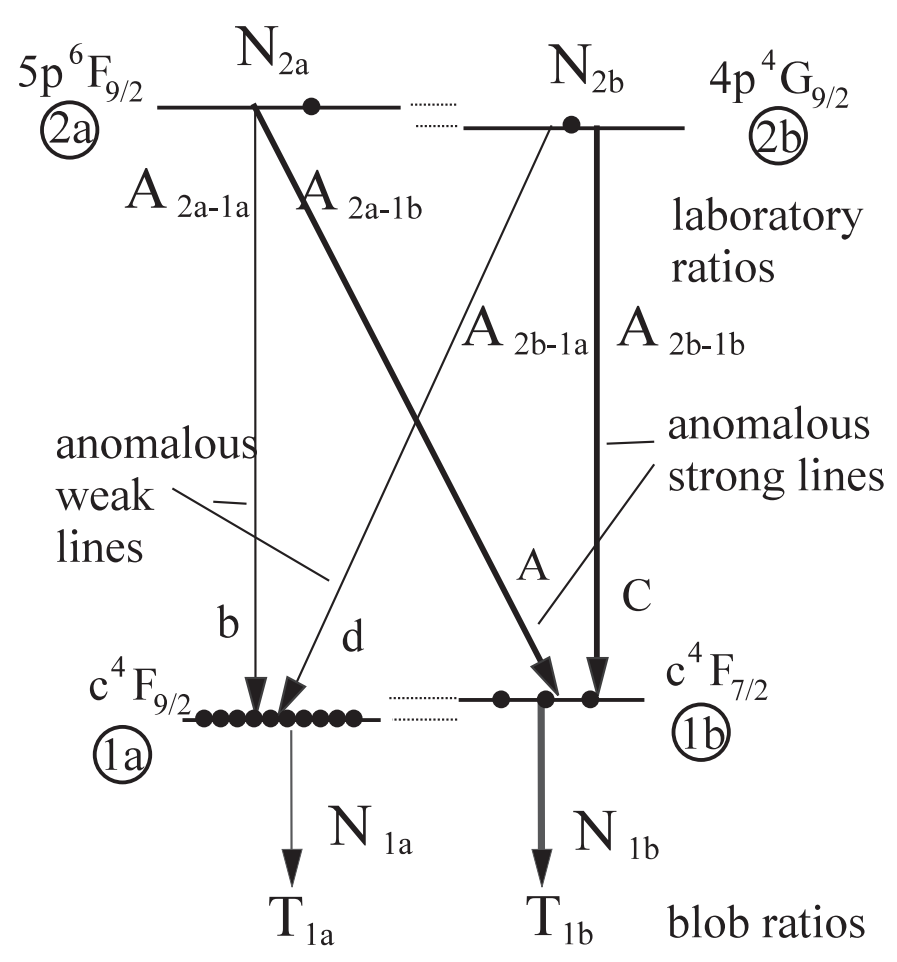

$$
\begin{array}{ll}
\lambda_{\mathrm{A}}=2507.55 \AA & \lambda_{\mathrm{C}}=2509.10 \AA \\
\lambda_{\mathrm{b}}=2505.64 \AA & \lambda_{\mathrm{d}}=2507.19 \AA
\end{array}
$$

Fig. 13. Detailed notation for the anomalous UV FeII spectral lines and the corresponding energy levels.

levels by the Ly $\alpha$ radiation (Johansson \& Letokhov $2001 \mathrm{~b}$ ). The rate of this process is too low to provide for any population inversion, but is quite sufficient to provide for the depopulation of the lower levels at a rate much higher than the radiative decay rate $1 / T_{1}$. According to Johansson \& Letokhov (2001b), for the effective temperature of $\operatorname{Ly} \alpha, T_{\text {eff }}(\mathrm{Ly} \alpha) \simeq 10000 \mathrm{~K}$, the resonance enhancement of the photoionization cross-section can provide for the depopulation of the lower states at a rate of $W_{\mathrm{ph}}^{1 \mathrm{i}} \gg 1 / T_{1}$. It is most important that the narrow resonances of the photoionization cross-sections of Fe II (Nahar \& Pradhan 1994) and the energy difference between the levels $\mathrm{c}^{4} \mathrm{~F}_{7 / 2}$ and $\mathrm{c}^{4} \mathrm{~F}_{9 / 2}$ (Fig. $4, \Delta E=30 \mathrm{~cm}^{-1}$ ) must provide for the preferable depopulation of the $c^{4} \mathrm{~F}_{7 / 2}$ state (1b) and the resulting weaker absorption of the A and $\mathrm{C}$ ultraviolet lines. According to (38), it is necessary that the $\sigma_{\mathrm{ph}}^{1 \mathrm{i}}$ ratio should be

$$
\frac{\sigma_{\mathrm{ph}}^{1 \mathrm{~b}, \mathrm{i}}}{\sigma_{\mathrm{ph}}^{1 \mathrm{a}, \mathrm{i}}} \simeq\left(\frac{I_{\mathrm{A}}}{I_{\mathrm{b}}}\right)^{\mathrm{blob}}\left(\frac{I_{\mathrm{A}}}{I_{\mathrm{b}}}\right)^{\mathrm{lab}} \simeq 30
$$

which can be proved by precise calculations similar to those reported in (Nahar \& Pradhan 1994) in the range slightly above the ionization limit of FeII. The requirement in Eq. (58) is rahter unusual for photoionization of levels belonging to the same LS term of an electron configuration. Perhaps, this contradiction can be overcome in the frame of plausible photodepletion of the $c^{4} \mathrm{~F}$ levels by relatively narrow autoionization levels (Johansson \& Letokhov 2001b, Fig. 9). However, this is subject to more detailed and elaborative atomic structure calculations and/or experiments, which are out of the scope of the present paper.

The estimates in Eq. (58) require the following value of $\kappa_{\mathrm{abs}}(\mathrm{UV})$ :

$\kappa_{\mathrm{abs}}^{(\mathrm{UV})} \simeq\left(10^{-2}-10^{-1}\right) \kappa_{\mathrm{sc}}^{(\mathrm{UV})}$.

This requirement must be compatible with the need for the $\operatorname{Ly} \alpha$ photons to be scattered many times, $\tau_{0} \simeq 10^{7}$, prior to their decay (Johansson \& Letokhov 2001b) to provide for a substantial diffusive broadening of the Ly $\alpha$ spectrum. The most suitable non-resonance absorption mechanism is the photoionization absorption of He I to long-lived metastable states that are known to accumulate in the passive $\mathrm{HI}$ region during the $\mathrm{HeI} / \mathrm{HeII} / \mathrm{He}^{*} \mathrm{I}$ photoionization/recombination cycle under the effect of the shorter-wavelength EUV radiation from the central star (Ambartzumian 1939). Let us assume that the continuous absorption coefficients $\kappa_{\text {abs }}$ are of the same order of magnitude for Ly $\alpha$ and UV FeII photons, i.e. $\kappa_{\mathrm{abs}}(\operatorname{Ly} \alpha)$. It follows from these crude estimates that the ratio between the resonance scattering coefficients for these two wavelengths is

$\frac{\kappa_{\mathrm{sc}}(\mathrm{Ly} \alpha)}{\kappa_{\mathrm{sc}}(\mathrm{UV})}=\frac{N_{\mathrm{HI}}}{N_{\mathrm{Fe} I \mathrm{II}}^{1}} \frac{\sigma_{0}(\mathrm{Ly} \alpha)}{\sigma_{12}(\mathrm{FeII})}=\frac{N_{\mathrm{HI}}}{N_{\mathrm{FeII}}} \frac{N_{\mathrm{FeII}}}{N_{\mathrm{FeII}}^{1}} \frac{\sigma_{0}(\mathrm{Ly} \alpha)}{\sigma_{12}(\mathrm{FeII})}$,

where $N_{\mathrm{HI}}$ and $N_{\mathrm{Fe}}$ are the hydrogen and iron concentrations in the blob, respectively. The factor $N_{\mathrm{FeII}}^{1}$ is the concentration of FeII ions in the long-lived state $1\left(c^{4} \mathrm{~F}\right)$, which governs the optical density of the $1 \rightarrow 2$ transition and the value is, according to the estimates presented in Johansson \& Letokhov (2001b), $N_{\mathrm{FeII}}^{1} \simeq 3 \times 10^{-3} N_{\mathrm{FeII}}^{m}$, where $m$ is the metastable state. If we take the standard estimate for $N_{\mathrm{HI}} / N_{\mathrm{FeII}} \simeq 10^{4}$, we get from relation (60)

$\kappa_{\mathrm{sc}}\left(\mathrm{Ly}_{\alpha}\right) \simeq 3 \times 10^{6} \kappa_{\mathrm{sc}}(\mathrm{UV})$,

and so requirement (59) corresponds to

$\kappa_{\mathrm{abs}}\left(\mathrm{Ly}_{\alpha}\right) \simeq \kappa_{\mathrm{abs}}(\mathrm{UV})=3\left(10^{-9}-10^{-8}\right) \kappa_{\mathrm{sc}}\left(\mathrm{Ly}_{\alpha}\right)$.

Such a weak absorption of the Ly $\alpha$ photons with their short scattering length is known to provide for the necessary number of their scattering events, $\tau_{0} \simeq 10^{7}$.

\section{Conclusion}

Within the framework of the qualitative model presented here, which is a natural development of the general model presented in (Johansson \& Letokhov 2001a), the anomalous intensity ratio between the close UV FeII spectral lines of a gas condensation (blob B) in the vicinity of the star $\eta$ Carinae can be explained without the need to introduce stimulated amplification. The model can further be verified by numerical calculatiions of narrow (a few tens or hundreds of $\mathrm{cm}^{-1}$ ) resonances of the photoionization 
of the FeII ion from the quantum states $c^{4} \mathrm{~F}_{7 / 2,9 / 2}$ near their photoionization limit. Finally, this work confirms the importance of the calculations by Hummer (1968) of the effect of continuous absorption on the observed intensity ratio between spectral lines in nebulae. Obviously, to create a quantitative model of the photoprocesses occurring in the gas condensations in the vicinity of $\eta$ Car, it is necessary to compute the resonance transfer of the Ly $\alpha$ radiation from the highly ionized HII region to the weakly ionized, optically denser HI region precisely in the inhomogeneous transition layer, i.e., in the neighborhood of the boundary of the Strömgren sphere.

Acknowledgements. One of the authors (V.L.) is very grateful to the Swedish Natural Science Research Council (NFR) for the T. Erlander Professorship-2000, which allows him to explore the "laser-like" atomic physics with Hubble Space Telescope data in the stimulating atmosphere of Lund University. The project is also supported by grants (S.J.) from the Swedish National Space Board and the Crafoord Foundation. The authors are also grateful to the anonymous referee for valuable comments and criticism.

\section{References}

Abramovitz, M., \& Stegun, I. A. 1964, Handbook of Math. Functions (National Bureau of Standards, Wash. DC)

Adams, T. F. 1972, ApJ, 174, 439

Ambartzumian, V. A. 1939. Theoretical Astrophysics (Gosteklizdat, Moscow)

Avrett, D., \& Hummer, D. 1965, MNRAS, 130, 295

Courant, R., \& Hilbert, D. 1962, Methods of Mathematical Physics (Wiley-InterScience, New York), 1

Davidson, K., \& Humphreys, R. M. 1997, ARA\&A, 35, 1

Davidson, K. 1999, in $\eta$ Carinae at the Millenium, ed. J. A. Morse, R. M. Humphreys, \& A. Damineli, ASP Conf. Ser., 179,6
Frisch, H. 1980, A\&A, 87, 357

Hamann, F., Davidson, K., Ishibashi, K., \& Gull, T. R. 1999, in $\eta$ Carinae at the Millenium, ASP Conf. Ser., 179, 116

Harrington, J. P. 1973, MNRAS, 162, 43

Hummer, D. 1968, MNRAS, 138, 73

Hummer, D. G., \& Kunasz, P. B. 1980, ApJ, 236, 609

Ivanov, V. V. 1973, Transfer of Radiation in Spectral Lines, NBS SP-285, US Dept. of Commerce (Washington, D. C.) (translated by Hummer, D. G. and Weppner, E.)

Johansson, S., Davidson, K., Ebbets, D., et al. 1996, in Science with Hubble Telescope-II, ed. P. Benvenuti, F. D. Macehetto, \& F. J. Schreier, Space Telescope Institute, 361

Johansson, S., \& Letokhov, V. S. 2001a, in $\eta$ Carinae and Other Mysterious Stars, ed. T. R. Gull, S. Johansson, \& K. Davidson, ASP Conf. Ser., 242, 309

Johansson, S., \& Letokhov, V. S. 2001b, A\&A, 378, 266

Johansson, S., \& Zethson, T. 1999, in $\eta$ Carinae at the Millenium, ed. J. A. Morse, R. M. Humphreys, \& A. Damineli, ASP Conf. Ser., 179, 171

Kalitkin, N. N. 1978, Numerical Methods (Russian) (Nauka Publ. House, Moscow)

Kurucz, R. L. 1988, Trans. IAOXXB, ed. M. NcNally (Kluwer, Dordrecht), 168

Mihalas, D. 1978, Stellar Atmospheres (W. H. Freeman and Company, New York)

Nahar, S. N., \& Pradhan, A. K. 1994, J. Phys. B: At. Mol. Opt. Phys., 27, 429

Osterbrock, D. E. 1962, ApJ, 135, 195

Sobolev, V. V. 1949a, AJ (Russia) 26, 129; (1954) 31, 231

Sobolev, V. V. 1949b, Astr. Zh., 26, 129; 1951. Astr. Zh., 28, 355; 1954. Astr. Zh., 31, 231; 1963. A Treatise on Radiative Transfer (translated from Russian) van Nostrand, Princeton, N.J.

Svelto, O. 1989, Principles of Lasers (Plenum Press, New York and London)

Weigelt, G., \& Ebersberger, J. 1986, A\&A, 169, 65 